\title{
A thick-point approximation of a small body embedded in an elastic medium: justification with an asymptotic analysis
}

\author{
Arnaud Heibig* $\quad$ Nidhal Mannai*† Adrien Petrov* $\quad$ Yves Renard*
}

June 29, 2020

\begin{abstract}
The present paper proposes an approximation of the influence of a small inclusion, having different stiffness, in an elastic medium restricting its cinematics to a finite dimensional space. The problem, for the unknown displacement, is written in accordance of transmission conditions between elastic medium and inclusion. The multi-scale asymptotic expansions techniques, by using some corrective terms, are introduced to model the inclusion influence in both initial and approximated problems. Finally, some error estimates for asymptotic expansions are presented and different strategies are compared.
\end{abstract}

Key words. Asymptotic expansion, linear elasticity, multi-scale analysis, transmission problem, solvability

\section{Introduction}

This paper introduces a new approximation of the influence of a small inclusion in an elastic medium, so-called thick-point approximation, consisting in a restriction of cinematics of the inclusion to some predefined finite dimensional spaces such as spaces of degree one or two polynomials. A rigorous justification is given in terms of asymptotic analysis allowing to quantify the approximation order as well.

The interface problems served in the last decades as a cornerstone for developing mathematical models for inclusions embedded in elastic media. Some of these models are used in automotive industry to design tires having specific structure stiffnesses. A considerable amount of engineering and mathematical literature is devoted to this topic. We are specially interested in small elastic inclusions or inhomogeneities in a two-dimensional elastic body. Since the inclusions are usually small compared to the size of the body, it is rather costly from numerical viewpoint to take them into account. Naturally, the numerical approximation of such problems requires a severe mesh refinement near the inclusions, which sometimes prevents from taking them into account in the computations. If the inclusions are arranged within a

\footnotetext{
*Université de Lyon, CNRS, INSA de Lyon Institut Camille Jordan UMR 5208, 20 Avenue A. Einstein, F-69621 Villeurbanne, France (arnaud.heibig@insa-lyon.fr, nidhal.mannai@insa-lyon.fr, apetrov@math.univ-lyon1.fr, yves.renard@insa-lyon.fr)

${ }^{\dagger}$ Laboratoire de Modélisation Mathématique et Numérique dans les Sciences de l'Ingénieur, Ecole Nationale d'Ingénieurs de Tunis (ENIT), Université de Tunis El Manar, Campus Universitaire Farhat Hached, B.P. n 94 - ROMMANA, Tunis 106, Tunisia
} 
periodic network, the homogenization techniques could be used and the macroscopic model is valid, provided the characteristic properties of the body are modified accordingly (see [1,23]). Such approaches do not hold for local inhomogeneities, which are usually omitted in the applications, at least for the smallest ones, and/or integrated into the macroscopic domain. An other approach consists to use the asymptotic analysis to determine the influence of inclusions (see $[7,10,16,17,29,32]$ ) which is adopted in the present work.

Many references in the literature deal with the case of perturbations whether transmission problems (essentially for conductivity and Helmholtz equation) and perforated domains and cavities. For further details, the reader is referred to $[29,18,7,26,27,22,17,10,11]$ and to the reference therein. Concerning the case of inclusions, a method allowing to find the stresses set up in an elastic body was originally introduced in [13] and later on developed in [14, 28, 19, 20, 4, 30, 25] for various inclusions shape. On the other hand, the reconstruction techniques of small inclusions using only boundary measurements are described in [2]. The approach used in this work consists in deriving some asymptotic expansions of the boundary perturbations coming from the inclusions influences that has been initiated in [5] and extended in this work to the approximated case up to the order second order leading to deduce some approximation error estimates. Some other contributions dealing with the techniques using asymptotic expansions to treat inclusions can be found in $[31,6]$.

This paper is organized as follows. In Section 2, we give precisely the geometrical setting we shall work within. More precisely, the solvability of a pair of two dimensional approximations of the transmission problem between a body and inclusion both supposed to be elastic is recalled. Then Section 3 emphasizes some optimal error estimates for asymptotic expansions. Section 4 is dedicated to describe the thick-point approximation and perform its asymptotic expansion, up to the second order. This allows in Section 5 to give some error estimates depending on the choice of the finite dimensional space used. Section 6 treats the special case of a circular inclusion obtaining some optimal results. Section 7 gives the strong form of the thick-point approximation problem when first degree polynomial are used. Finally, a few conclusions are given in Section 8.

\section{Mechanical model and mathematical formulation}

We consider a reference configuration $\Omega \subset \mathbb{R}^{2}$ supposed to be with a Lipschitz-continuous boundary $\Gamma$. Let $\Omega_{f}^{\varepsilon} \subset \mathbb{R}^{2}$ be a bounded connected domain of characteristic dimension $\varepsilon$ representing an inclusion in $\Omega$. The boundary of this inclusion is denoted by $\Gamma^{\varepsilon}$ and $\Omega_{f}^{1} \stackrel{\text { def }}{=} \frac{\Omega_{f}^{\varepsilon}}{\varepsilon}$. The inclusion is assumed to be small enough compared to the characteristic size of the matrix represented by $\Omega_{m}^{\varepsilon} \stackrel{\text { def }}{=} \Omega \backslash \bar{\Omega}_{f}^{\varepsilon} \subset \mathbb{R}^{2}$ of radius $R$ with a Lipschitz-continuous $\Gamma^{\varepsilon}$ (see FIGURE 1 .

The present work deals with an antiplane problem which can be reduced to a Poisson problem. More precisely, we focus on the normal component to the plane $\left(O, x_{1}, x_{2}\right)$ denoted by $\widetilde{u}^{\varepsilon}\left(x_{1}, x_{2}\right)$. We assume the non-homogenous Dirichlet conditions and the continuity conditions on the boundary $\Gamma^{\varepsilon}$ hold on $\Gamma$ and $\Gamma^{\varepsilon}$, respectively. Let $\mathrm{U} \in \mathrm{H}^{1 / 2}(\Gamma)$ be a prescribed displacement on $\Gamma$. The mathematical problem is 


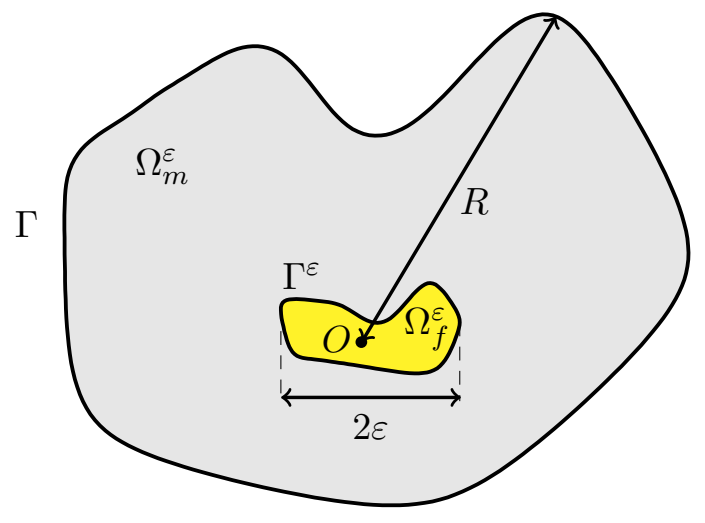

Figure 1: A small inclusion in an elastic medium

formulated as follows:

$$
\begin{array}{r}
-\alpha_{f} \Delta \widetilde{u}_{f}^{\varepsilon}=0 \text { in } \Omega_{f}^{\varepsilon}, \\
-\alpha_{m} \Delta \widetilde{u}_{m}^{\varepsilon}=0 \text { in } \Omega_{m}^{\varepsilon}, \\
\widetilde{u}_{f}^{\varepsilon}=\widetilde{u}_{m}^{\varepsilon} \text { on } \Gamma^{\varepsilon}, \\
\alpha_{f} \partial_{\mathbf{n}} \widetilde{u}_{f}^{\varepsilon}=\alpha_{m} \partial_{\mathbf{n}} \widetilde{u}_{m}^{\varepsilon} \text { on } \Gamma^{\varepsilon}, \\
\widetilde{u}_{m}^{\varepsilon}=\mathrm{U} \text { on } \Gamma,
\end{array}
$$

where $\partial_{\mathbf{n}}$ denote the normal derivative, $\alpha_{f}>0$ and $\alpha_{m}>0$ are the shear coefficients in the inclusion and in the matrix, respectively while $\widetilde{u}_{f}^{\varepsilon}$ and $\widetilde{u}_{m}^{\varepsilon}$ are the unknown solutions to problem (2.1) in the inclusion $\Omega_{f}^{\varepsilon}$ and in the matrix $\Omega_{m}^{\varepsilon}$, respectively.

Let us describe the weak formulation associated to problem (2.1). Introducing the set

$$
\widetilde{\mathrm{V}}_{\mathrm{U}} \stackrel{\text { def }}{=}\left\{v \in \mathrm{H}^{1}(\Omega): v_{\left.\right|_{\Gamma}}=\mathrm{U}\right\}
$$

the weak formulation associated to problem (2.1) is given by

$$
\left\{\begin{array}{l}
\text { find } \widetilde{u}^{\varepsilon} \in \widetilde{\mathrm{V}}_{\mathrm{U}} \text { such that for all } w \in \mathrm{H}_{0}^{1}(\Omega) \\
\int_{\Omega_{f}^{\varepsilon}} \alpha_{f} \nabla \widetilde{u}_{f}^{\varepsilon} \cdot \nabla w_{f} \mathrm{~d} x+\int_{\Omega_{m}^{\varepsilon}} \alpha_{m} \nabla \widetilde{u}_{m}^{\varepsilon} \cdot \nabla w_{m} \mathrm{~d} x=0 .
\end{array}\right.
$$

Since $\mathrm{U} \in \mathrm{H}^{1 / 2}(\Gamma)$, the trace theorem implies that there exists $g \in \mathrm{H}^{1}(\Omega)$ satisfying $g_{\left.\right|_{\Gamma}}=\mathrm{U}$. Let us define $\widetilde{u}_{0}^{\varepsilon} \in \mathrm{H}_{0}^{1}(\Omega)$ such that $\widetilde{u}^{\varepsilon} \stackrel{\text { def }}{=} \widetilde{u}_{0}^{\varepsilon}+g \in \widetilde{\mathrm{V}}_{\mathrm{U}}$. Hence, the weak formulation (2.2) can be rewritten as follows

$$
\left\{\begin{array}{l}
\text { find } \widetilde{u}_{0}^{\varepsilon} \in \mathrm{H}_{0}^{1}(\Omega) \text { such that for all } w \in \mathrm{H}_{0}^{1}(\Omega), \\
\int_{\Omega_{f}^{\varepsilon}} \alpha_{f} \nabla \widetilde{u}_{0 f}^{\varepsilon} \cdot \nabla w_{f} \mathrm{~d} x+\int_{\Omega_{m}^{\varepsilon}} \alpha_{m} \nabla \widetilde{u}_{0 m}^{\varepsilon} \cdot \nabla w_{m} \mathrm{~d} x \\
=-\int_{\Omega_{f}^{\varepsilon}} \alpha_{f} \nabla g \cdot \nabla w_{f} \mathrm{~d} x-\int_{\Omega_{m}^{\varepsilon}} \alpha_{m} \nabla g \cdot \nabla w_{m} \mathrm{~d} x .
\end{array}\right.
$$

The existence and uniqueness results to problem (2.3) can be proved by using Lax-Milgram's theorem. 


\section{Asymptotic expansion for the antiplane problem}

This section is devoted to the asymptotic expansion of the antiplane problem up to second order. The reader is referred to [5] for further details on this approach. This step plays a crucial role to get some error estimates with respect to the size of the inclusion for the approximation.

\subsection{First order expansion for the antiplane problem}

We consider first the asymptotic expansion of the first order. To this aim, we define by $\widetilde{u}^{0}$ the displacement in $\Omega$ without any inclusion. Taking $\varepsilon=0$ in (2.2), we get the following weak formulation

$$
\left\{\begin{array}{l}
\text { find } \widetilde{u}^{0} \in \widetilde{\mathrm{V}}_{\mathrm{U}} \text { such that for all } w \in \mathrm{H}_{0}^{1}(\Omega), \\
\int_{\Omega} \alpha_{m} \nabla \widetilde{u}^{0} \cdot \nabla w \mathrm{~d} x=0 .
\end{array}\right.
$$

Substracting (2.2) to (3.1), we obtain

$$
\int_{\Omega_{f}^{\varepsilon}}\left(\alpha_{f}-\alpha_{m}\right) \nabla \widetilde{u}^{0} \cdot \nabla w_{f} \mathrm{~d} x+\int_{\Omega_{f}^{\varepsilon}} \alpha_{f} \nabla\left(\widetilde{u}_{f}^{\varepsilon}-\widetilde{u}^{0}\right) \cdot \nabla w_{f} \mathrm{~d} x+\int_{\Omega_{m}^{\varepsilon}} \alpha_{m} \nabla\left(\widetilde{u}_{m}^{\varepsilon}-\widetilde{u}^{0}\right) \cdot \nabla w_{m} \mathrm{~d} x=0
$$

for all $w \in \mathrm{H}_{0}^{1}(\Omega)$. By using the following notation $\widetilde{\mathrm{d}}_{k}^{0 \varepsilon} \stackrel{\text { def }}{=} \widetilde{u}_{k}^{\varepsilon}-\widetilde{u}^{0}, k \in\{f, m\}$, the identity (3.2) can be rewritten as follows:

$$
\int_{\Omega_{f}^{\varepsilon}}\left(\alpha_{f}-\alpha_{m}\right) \nabla \widetilde{u}^{0} \cdot \nabla w_{f} \mathrm{~d} x+\int_{\Omega_{f}^{\varepsilon}} \alpha_{f} \nabla \widetilde{\mathrm{d}}_{f}^{0 \varepsilon} \cdot \nabla w_{f} \mathrm{~d} x+\int_{\Omega_{m}^{\varepsilon}} \alpha_{m} \nabla \widetilde{\mathrm{d}}_{m}^{0 \varepsilon} \cdot \nabla w_{m} \mathrm{~d} x=0
$$

for all $w \in \mathrm{H}_{0}^{1}(\Omega)$. In order to deal with fixed domain and interface, we introduce the following scaling $y \stackrel{\text { def }}{=} \frac{x}{\varepsilon}$ and we define $\widetilde{\mathrm{D}}_{k}^{0 \varepsilon}(y) \stackrel{\text { def }}{=} \widetilde{\mathrm{d}}_{k}^{0 \varepsilon}(x), k \in\{f, m\}$. It comes that

$$
\begin{aligned}
& \varepsilon \int_{\Omega_{f}^{1}}\left(\alpha_{f}-\alpha_{m}\right) \nabla \widetilde{u}^{0}(\varepsilon y) \cdot \nabla w_{f}(\varepsilon y) \mathrm{d} y+\int_{\Omega_{f}^{1}} \alpha_{f} \nabla \widetilde{\mathrm{D}}_{f}^{0 \varepsilon}(y) \cdot \nabla w_{f}(\varepsilon y) \mathrm{d} y \\
& +\int_{\Omega_{m}^{\varepsilon} / \varepsilon} \alpha_{m} \nabla \mathrm{D}_{m}^{0 \varepsilon}(y) \cdot \nabla w_{m}(\varepsilon y) \mathrm{d} y=0
\end{aligned}
$$

for all $w \in \mathrm{H}_{0}^{1}(\Omega)$. Let $\Omega^{\infty} \stackrel{\text { def }}{=} \mathbb{R}^{2} \backslash \Omega_{f}^{1}$ and $\widetilde{\mathrm{V}}_{\text {log }}$ be an Hilbert space defined by

$$
\widetilde{\mathrm{V}}_{\log } \stackrel{\text { def }}{=}\left\{v \in \mathcal{D}^{\prime}\left(\mathbb{R}^{2}\right):\left(1+|x|^{2}\right)^{-\frac{1}{2}}\left(\log \left(2+|x|^{2}\right)\right)^{-1} v \in \mathrm{L}^{2}\left(\mathbb{R}^{2}\right) \text { and } \nabla v \in \mathrm{L}^{2}\left(\mathbb{R}^{2}\right)^{2}\right\},
$$

endowed with the norm

$$
\|u\|_{\widetilde{\mathrm{V}}_{\log }} \stackrel{\text { def }}{=}\left(\left\|\left(1+|x|^{2}\right)^{-\frac{1}{2}}\left(\log \left(2+|x|^{2}\right)\right)^{-1} v\right\|_{\mathrm{L}^{2}\left(\mathbb{R}^{2}\right)}^{2}+\|\nabla v\|_{\left.\mathrm{L}\left(\mathbb{R}^{2}\right)\right)^{2}}^{2}\right)^{\frac{1}{2}}
$$

The reader is referred to [5, Appendix] for some further explanations on this functional space. Fix $R^{0} \gg 1$, we define a closed subspace $\widetilde{\mathrm{V}}_{\log }^{R^{0}} \hookrightarrow \widetilde{\mathrm{V}}_{\log }$ by

$$
\widetilde{\mathrm{V}}_{\log }^{R^{0}} \stackrel{\text { def }}{=}\left\{v \in \widetilde{\mathrm{V}}_{\log }: \int_{-\pi}^{\pi} v\left(R^{0} \cos (\theta), R^{0} \sin (\theta)\right) \mathrm{d} \theta=0\right\} .
$$


We assume $\varepsilon$ small enough compared to the size of $\Omega$ and we eliminate completely the dependence on $\varepsilon$ by approximating the identity (3.4) as follows:

$$
\left\{\begin{array}{l}
\text { find } \widetilde{W}^{0} \in \widetilde{\mathrm{V}}_{\log }^{R^{0}} \text { such that for all } w \in \widetilde{\mathrm{V}}_{\log }^{R^{0}}, \\
\int_{\Omega_{f}^{1}}\left(\alpha_{f}-\alpha_{m}\right) \nabla \widetilde{u}^{0}(0) \cdot \nabla w_{f} \mathrm{~d} y+\int_{\Omega_{f}^{1}} \alpha_{f} \nabla \widetilde{W}_{f}^{0} \cdot \nabla w_{f} \mathrm{~d} y+\int_{\Omega^{\infty}} \alpha_{m} \nabla \widetilde{W}_{m}^{0} \cdot \nabla w_{m} \mathrm{~d} y=0
\end{array}\right.
$$

Note that all the constant functions are included in $\widetilde{V}_{\log }$ and Poincaré's inequality can be obtained on the quotient space of the weighted Sobolev space with constant functions. Clearly, the last two terms on the left hand side of (3.5) are bilinear, coercive and continuous forms on $\widetilde{V}_{\log }^{R^{0}}$ while the first term on the term on the left hand side of (3.5) is linear and continuous on $\widetilde{V}_{\log }^{R^{0}}$. Then, the existence and uniqueness results come from Lax-Milgram's theorem. On the other hand, we observe that

$$
\begin{aligned}
& \int_{\Omega_{f}^{\varepsilon}}\left(\alpha_{f}-\alpha_{m}\right) \nabla \widetilde{u}^{(0)}(0) \cdot \nabla w_{f} \mathrm{~d} x+\int_{\Omega_{f}^{\varepsilon}} \alpha_{f} \nabla\left(\varepsilon \widetilde{W}_{f}^{0}\left(\frac{\dot{-}}{\varepsilon}\right)\right) \cdot \nabla w_{f} \mathrm{~d} x \\
& +\int_{\Omega_{m}^{\varepsilon}} \alpha_{m} \nabla\left(\varepsilon \widetilde{W}_{m}^{0}\left(\frac{\dot{-}}{\varepsilon}\right)\right) \cdot \nabla w_{m} \mathrm{~d} x=0
\end{aligned}
$$

for all $w \in \mathrm{H}_{0}^{1}(\Omega)$. Subtracting (3.2) to (3.6), we find

$$
\begin{aligned}
& \int_{\Omega_{f}^{\varepsilon}}\left(\alpha_{f}-\alpha_{m}\right)\left(\nabla \widetilde{u}^{(0)}-\nabla \widetilde{u}^{(0)}(0)\right) \cdot \nabla w_{f} \mathrm{~d} x+\int_{\Omega_{f}^{\varepsilon}} \alpha_{f} \nabla\left(\widetilde{u}-\widetilde{u}^{(0)}-\varepsilon \widetilde{W}^{0}\left(\frac{\dot{\varepsilon}}{\varepsilon}\right)\right) \cdot \nabla w_{f} \mathrm{~d} x \\
& +\int_{\Omega_{m}^{\varepsilon}} \alpha_{m} \nabla\left(\widetilde{u}-\widetilde{u}^{(0)}-\varepsilon \widetilde{W}^{0}\left(\frac{\dot{\varepsilon}}{\varepsilon}\right)\right) \cdot \nabla w_{m} \mathrm{~d} x=0
\end{aligned}
$$

for all $w \in \mathrm{H}_{0}^{1}(\Omega)$. Setting $\widetilde{\mathrm{d}}^{1 \varepsilon} \stackrel{\text { def }}{=} \widetilde{u}^{\varepsilon}-\widetilde{u}^{0}-\varepsilon \widetilde{W}^{0}\left(\frac{\cdot}{\varepsilon}\right)$, with $x \in \Omega$, we also have

$$
\left\{\begin{array}{l}
\widetilde{\mathrm{d}}^{1 \varepsilon} \in \mathrm{H}^{1}(\Omega) \text { and for all } w \in \mathrm{H}_{0}^{1}(\Omega), \\
\int_{\Omega_{f}^{\varepsilon}}\left(\alpha_{f}-\alpha_{m}\right)\left(\nabla \widetilde{u}^{(0)}-\nabla \widetilde{u}^{(0)}(0)\right) \cdot \nabla w_{f} \mathrm{~d} x+\int_{\Omega_{f}^{\varepsilon}} \alpha_{f} \nabla \widetilde{\mathrm{d}}^{1 \varepsilon} \cdot \nabla w_{f} \mathrm{~d} x \\
+\int_{\Omega_{m}^{\varepsilon}} \alpha_{m} \nabla \widetilde{\mathrm{d}}^{1 \varepsilon} \cdot \nabla w_{m} \mathrm{~d} x=0, \\
\text { with } \widetilde{\mathrm{d}}^{1 \varepsilon}=-\varepsilon \widetilde{W}^{0}\left(\frac{\cdot}{\varepsilon}\right) \text { on } \Gamma .
\end{array}\right.
$$

The following estimate on the rest $\widetilde{\mathrm{d}}^{1 \varepsilon}$ of the first order expansion can be proved.

Lemma 3.1. There exists a constant $C>0$, independent of $\varepsilon$, such that

$$
\left\|\widetilde{\mathrm{d}}^{1 \varepsilon}\right\|_{\mathrm{H}^{1}(\Omega)} \leq C \varepsilon^{2} .
$$

Proof. Let $\mathcal{L}: \mathrm{H}^{1 / 2}(\Gamma) \rightarrow \mathrm{H}^{1}(\Omega)$ be a continuous lifting operator [24]. We define $\widetilde{z}^{\varepsilon} \stackrel{\text { def }}{=} \mathcal{L}\left(-\varepsilon \widetilde{W}^{0}(\dot{\bar{\varepsilon}})\right)$ and 
$\widetilde{r}^{1 \varepsilon} \stackrel{\text { def }}{=} \widetilde{\mathrm{d}}^{1 \varepsilon}-\widetilde{z}^{\varepsilon}$. Clearly, $\widetilde{r}^{1 \varepsilon} \in \mathrm{H}_{0}^{1}(\Omega)$ and according to (3.8), we get

$$
\begin{aligned}
& \int_{\Omega_{f}} \alpha_{f} \nabla \widetilde{r}_{f}^{1 \varepsilon} \cdot \nabla w_{f} \mathrm{~d} x+\int_{\Omega_{m}} \alpha_{m} \nabla \widetilde{r}_{m}^{1 \varepsilon} \cdot \nabla w_{m} \mathrm{~d} x+\int_{\Omega_{f}^{\varepsilon}}\left(\alpha_{f}-\alpha_{m}\right)\left(\nabla \widetilde{u}^{0}(0)-\nabla \widetilde{u}^{0}\right) \cdot \nabla w_{f} \mathrm{~d} x \\
& =-\int_{\Omega_{f}} \alpha_{f} \nabla \widetilde{z^{\varepsilon}} \cdot \nabla w_{f} \mathrm{~d} x-\int_{\Omega_{m}} \alpha_{m} \nabla \widetilde{z^{\varepsilon}} \cdot \nabla w_{m} \mathrm{~d} x .
\end{aligned}
$$

Taking $w=\widetilde{r}^{1 \varepsilon} \in \mathrm{H}_{0}^{1}(\Omega)$, we find

$$
\begin{aligned}
& \alpha_{f}\left\|\nabla \widetilde{r}_{f}^{1 \varepsilon}\right\|_{L^{2}\left(\Omega_{f}^{\varepsilon}\right)}^{2}+\alpha_{m}\left\|\nabla \widetilde{r}_{m}^{1 \varepsilon}\right\|_{L^{2}\left(\Omega_{m}^{\varepsilon}\right)}^{2}+\int_{\Omega_{f}^{\varepsilon}}\left(\alpha_{f}-\alpha_{m}\right)\left(\nabla \widetilde{u}^{0}(0)-\nabla \widetilde{u}^{0}\right) \cdot \nabla \widetilde{r}_{f}^{1 \varepsilon} \mathrm{d} x \\
& =-\int_{\Omega_{f}^{\varepsilon}} \alpha_{f} \nabla \widetilde{z^{\varepsilon}} \cdot \nabla \widetilde{r}_{f}^{1 \varepsilon} \mathrm{d} x-\int_{\Omega_{m}^{\varepsilon}} \alpha_{m} \nabla \widetilde{z^{\varepsilon}} \cdot \nabla \widetilde{r}_{m}^{1 \varepsilon} \mathrm{d} x,
\end{aligned}
$$

which by using Cauchy-Schwarz's inequality implies that

$$
\left\|\nabla \widetilde{r}^{1 \varepsilon}\right\|_{L^{2}(\Omega)} \leq C\left(\left\|\nabla \widetilde{u}^{0}(0)-\nabla \widetilde{u}^{0}\right\|_{L^{2}\left(\Omega_{f}^{\varepsilon}\right)}+\left\|\nabla \widetilde{z^{\varepsilon}}\right\|_{L^{2}(\Omega)}\right) .
$$

By Poincaré's inequality, we get

$$
\left\|\widetilde{r}^{1 \varepsilon}\right\|_{\mathrm{H}^{1}(\Omega)} \leq C\left(\left\|\nabla \widetilde{u}^{0}(0)-\nabla \widetilde{u}^{0}\right\|_{L^{2}\left(\Omega_{f}^{\varepsilon}\right)}+\left\|\nabla \widetilde{z^{\varepsilon}}\right\|_{L^{2}(\Omega)}\right) .
$$

Since $\widetilde{r}^{1 \varepsilon} \stackrel{\text { def }}{=} \widetilde{\mathrm{d}}^{1 \varepsilon}-\widetilde{z^{\varepsilon}}$, we obtain

$$
\left\|\widetilde{\mathrm{d}}^{1 \varepsilon}\right\|_{\mathrm{H}^{1}(\Omega)} \leq C\left(\left\|\nabla \widetilde{u}^{0}(0)-\nabla \widetilde{u}^{0}\right\|_{\mathrm{L}^{2}\left(\Omega_{f}^{\varepsilon}\right)}+\left\|{\widetilde{z^{\varepsilon}}}\right\|_{\mathrm{H}^{1}(\Omega)}\right) .
$$

By using the continuity of the lifting operator $\mathcal{L}$, it comes that

$$
\left\|\widetilde{\mathrm{d}}^{1 \varepsilon}\right\|_{\mathrm{H}^{1}(\Omega)} \leq C\left(\left\|\nabla \widetilde{u}^{0}(0)-\nabla \widetilde{u}^{0}\right\|_{\mathrm{L}^{2}\left(\Omega_{f}^{\varepsilon}\right)}+\varepsilon\left\|\widetilde{W}\left(\frac{\dot{-}}{\varepsilon}\right)\right\|_{\mathrm{H}^{1 / 2}(\Gamma)}\right) .
$$

We evaluate first $\left\|\nabla \widetilde{u}^{0}(0)-\nabla \widetilde{u}^{0}\right\|_{L^{2}\left(\Omega_{f}^{\varepsilon}\right)}$. To this aim, we observe that

$$
\nabla \widetilde{u}^{0}(x)=\nabla \widetilde{u}^{0}(0)+\mathcal{O}(|x|) .
$$

Then, we conclude that

$$
\left\|\nabla \widetilde{u}^{0}(x)-\nabla \widetilde{u}^{0}(0)\right\|_{L^{2}\left(\Omega_{f}^{\varepsilon}\right)} \leq|x| \leq C \varepsilon^{2} .
$$

It remains to evaluate $\|\widetilde{W}(\dot{\bar{\varepsilon}})\|_{\mathrm{H}^{1 / 2}(\Gamma)}$. Let us define $x \stackrel{\text { def }}{=}(r \cos (\theta), r \sin (\theta))$ for all $r \geq R^{0}$. Notice that $\widetilde{W}^{0} \in \widetilde{\mathrm{V}}_{\log }^{R^{0}}$ and there exists $\left(\widetilde{a}_{n}, \widetilde{b}_{n}\right) \in \mathbb{R}^{2}$ for all $n \in \mathbb{N}^{*}$ and $\theta \in[0,2 \pi]$ such that

$$
\widetilde{W}^{0}\left(\frac{\dot{\varepsilon}}{\varepsilon}\right)=\sum_{n \geq 1}\left(\frac{\varepsilon R^{0}}{r}\right)^{n}\left(\widetilde{a}_{n} \cos (n \theta)+\widetilde{b}_{n} \sin (n \theta)\right) .
$$


Let $\Omega_{\mathrm{c}}$ be a regular subset of $\mathbb{R}^{2}$ such that $\Gamma \in \stackrel{\Omega}{\mathrm{c}}_{\mathrm{c}}$ and there exist $\left(r_{1}, r_{2}\right) \in \mathbb{R}^{+} \times \mathbb{R}^{+}$satisfying $r_{1}<r<r_{2}$ on $\Omega_{\mathrm{c}}$. Then we infer that

$$
\left\|\widetilde{W}^{0}\left(\begin{array}{c}
\cdot \\
\varepsilon
\end{array}\right)\right\|_{\mathrm{H}^{1 / 2}(\Gamma)} \leq C\left\|\widetilde{W}^{0}\left(\begin{array}{c}
\cdot \\
\varepsilon
\end{array}\right)\right\|_{\mathrm{H}^{1}\left(\Omega_{\mathrm{c}}\right)} .
$$

Choosing $\varepsilon>0$ such that $\varepsilon<\frac{r_{1}}{2 R^{0}}$, we obtain

$$
\begin{aligned}
& \left\|\nabla \widetilde{W}^{0}\left(\frac{\cdot}{\varepsilon}\right)\right\|_{L^{2}\left(\Omega_{\mathrm{c}}\right)} \leq \frac{4 \varepsilon R^{0} \sqrt{\mu\left(\Omega_{\mathrm{c}}\right)}}{r_{1}^{2}} \sum_{n \geq 1} n\left(\frac{\varepsilon R^{0}}{r_{1}}\right)^{n-1}\left(\left|\widetilde{a}_{n}\right|+\left|\widetilde{b}_{n}\right|\right) \leq C \varepsilon, \\
& \left\|\widetilde{W}^{0}\left(\frac{\cdot}{\varepsilon}\right)\right\|_{L^{2}\left(\Omega_{\mathrm{c}}\right)} \leq \frac{2 \varepsilon R^{0}}{r_{1}} \sum_{n \geq 1}\left(\frac{\varepsilon R^{0}}{r_{1}}\right)^{n-1}\left(\left|\widetilde{a}_{n}\right|+\left|\widetilde{b}_{n}\right|\right) \leq C \varepsilon
\end{aligned}
$$

where $\mu\left(\Omega_{f}^{\varepsilon}\right)$ stands for the usual measure of $\Omega_{f}^{\varepsilon}$. Carrying (3.12) into (3.11), we get

$$
\left\|\widetilde{W}^{0}\left(\begin{array}{c}
\cdot \\
\varepsilon
\end{array}\right)\right\|_{\mathrm{H}^{1 / 2}(\Gamma)} \leq C \varepsilon
$$

Finally, we have

$$
\left\|\widetilde{\mathrm{d}}^{1 \varepsilon}\right\|_{\mathrm{H}^{1}(\Omega)} \leq C \varepsilon^{2}
$$

which concludes the proof.

\subsection{Second order expansion for the antiplane problem}

The approach adopted here is similar to the one used to obtain the first order expansion. Namely, let $\widetilde{\mathrm{D}}^{1 \varepsilon}(y) \stackrel{\text { def }}{=} \widetilde{\mathrm{d}}^{1 \varepsilon}(x)$ and observe that

$$
\begin{aligned}
& \frac{1}{\varepsilon} \int_{\Omega_{f}^{1}}\left(\alpha_{f}-\alpha_{m}\right)\left(\nabla \widetilde{u}^{(0)}-\nabla \widetilde{u}^{(0)}(0)\right) \cdot \nabla w_{f} \mathrm{~d} y+\int_{\Omega_{f}^{1}} \alpha_{f} \nabla \widetilde{\mathrm{D}}^{1 \varepsilon} \cdot \nabla w_{f} \mathrm{~d} y \\
& +\int_{\frac{\Omega_{m}^{\varepsilon}}{\varepsilon}} \alpha_{m} \nabla \widetilde{\mathrm{D}}^{1 \varepsilon} \cdot \nabla w_{m} \mathrm{~d} y=0
\end{aligned}
$$

for all $w \in \mathrm{H}_{0}^{1}\left(\frac{\Omega}{\varepsilon}\right)$. Then (3.13) is approximated by the following problem:

$$
\left\{\begin{array}{l}
\text { find } \widetilde{W}^{1} \in \widetilde{\mathrm{V}}_{\log }^{R^{0}} \text { such that for all } w \in \widetilde{\mathrm{V}}_{\log }^{R^{0}}, \\
\int_{\Omega_{f}^{1}}\left(\alpha_{f}-\alpha_{m}\right) \mathcal{H}_{\widetilde{u}^{0}}(0) y \cdot \nabla w_{f} \mathrm{~d} y+\int_{\Omega_{f}^{1}} \alpha_{f} \nabla \widetilde{W}_{f}^{1} \cdot \nabla w_{f} \mathrm{~d} y+\int_{\Omega^{\infty}} \alpha_{m} \nabla \widetilde{W}_{m}^{1} \cdot \nabla w_{m} \mathrm{~d} y=0
\end{array}\right.
$$

where $\widetilde{W}^{1}$ and $\mathcal{H}_{\widetilde{u}^{0}}(0)$ denote the second order corrector term and Hessian matrix associated to $\widetilde{u}^{0}$ in $x=0$, respectively. Observe that for all $w \in \mathrm{H}_{0}^{1}(\Omega)$ extended by zero in $\mathbb{R}^{2} \backslash \Omega, w$ belongs to $\widetilde{\mathrm{V}}_{\text {log }}$. Then we have

$$
\begin{aligned}
& \int_{\Omega_{f}^{\varepsilon}}\left(\alpha_{f}-\alpha_{m}\right) \mathcal{H}_{\widetilde{u}^{0}}(0) x \cdot \nabla w_{f} \mathrm{~d} x+\int_{\Omega_{f}^{\varepsilon}} \varepsilon^{2}\left(\alpha_{f}-\alpha_{m}\right) \nabla \widetilde{v} \cdot \nabla w_{f} \mathrm{~d} x \\
& +\int_{\Omega_{m}^{\varepsilon}} \alpha_{m} \nabla\left(\varepsilon^{2} \widetilde{W}_{m}^{1}\left(\frac{\dot{-}}{\varepsilon}\right)\right) \cdot \nabla w_{m} \mathrm{~d} x+\int_{\Omega_{f}^{\varepsilon}} \alpha_{f} \nabla\left(\varepsilon^{2} \widetilde{W}_{f}^{1}\left(\frac{\dot{-}}{\varepsilon}\right)\right) \cdot \nabla w_{f} \mathrm{~d} x=0
\end{aligned}
$$


for all $w \in \mathrm{H}_{0}^{1}(\Omega)$. On the one hand, let $\widetilde{v} \in \mathrm{H}^{1}(\Omega)$ be a corrector term allowing to eliminate the first order term $\widetilde{W}^{0}$ on $\Gamma$ and satisfying

$$
\int_{\Omega} \alpha_{m} \nabla \widetilde{v} \cdot \nabla w \mathrm{~d} x=0
$$

for all $w \in \mathrm{H}_{0}^{1}(\Omega)$ with $\widetilde{v}=-\frac{R^{0}}{r}\left(\widetilde{a}_{1} \cos (\theta)+\widetilde{b}_{1} \sin (\theta)\right)$ on $\Gamma$. Setting $\widetilde{\mathrm{d}}^{2 \varepsilon} \stackrel{\text { def }}{=} \widetilde{u}^{\varepsilon}-\widetilde{u}^{0}-\varepsilon \widetilde{W}^{0}\left(\frac{\dot{-}}{\varepsilon}\right)-\varepsilon^{2} \widetilde{W}^{1}\left(\frac{\dot{-}}{\varepsilon}\right)+\varepsilon^{2} \widetilde{v}$. According to (3.8), (3.15) and (3.16), we find

$$
\left\{\begin{array}{l}
\widetilde{\mathrm{d}}^{2 \varepsilon} \in \mathrm{H}^{1}(\Omega) \text { and for all } w \in \mathrm{H}_{0}^{1}(\Omega), \\
\int_{\Omega_{f}^{\varepsilon}}\left(\alpha_{f}-\alpha_{m}\right)\left(\nabla \widetilde{u}^{(0)}-\nabla \widetilde{u}^{(0)}(0)-\mathcal{H}_{\widetilde{u}^{0}}(0) x+\varepsilon^{2} \nabla \widetilde{v}\right) \cdot \nabla w_{f} \mathrm{~d} x \\
+\int_{\Omega_{f}^{\varepsilon}} \alpha_{f} \nabla \widetilde{\mathrm{d}}^{2 \varepsilon} \cdot \nabla w_{f} \mathrm{~d} x+\int_{\Omega_{m}^{\varepsilon}} \alpha_{m} \nabla \widetilde{\mathrm{d}}^{2 \varepsilon} \cdot \nabla w_{m} \mathrm{~d} x=0, \\
\text { with } \widetilde{\mathrm{d}}^{2 \varepsilon}=-\varepsilon \widetilde{W}^{0}\left(\frac{\dot{-}}{\varepsilon}\right)-\varepsilon^{2} \widetilde{W}^{1}\left(\frac{\dot{\varepsilon}}{\varepsilon}\right)+\varepsilon^{2} \widetilde{v} \text { on } \Gamma .
\end{array}\right.
$$

Let us define

$$
\widetilde{z}^{2 \varepsilon} \stackrel{\text { def }}{=} \mathcal{L}\left(-\varepsilon \widetilde{W}^{0}\left(\frac{\dot{\varepsilon}}{\varepsilon}\right)-\varepsilon^{2} \widetilde{W}^{1}\left(\frac{\cdot}{\varepsilon}\right)-\varepsilon^{2} \frac{R^{0}}{r}\left(\widetilde{a}_{1} \cos (\theta)+\widetilde{b}_{1} \sin (\theta)\right)\right) \quad \text { and } \quad \widetilde{r}^{2 \varepsilon} \stackrel{\text { def }}{=} \widetilde{\mathrm{d}}^{2 \varepsilon}-\widetilde{z}^{2 \varepsilon}
$$

where $\mathcal{L}$ denotes the lifting operator introduced above. Similarly to the first order expansion, we may prove that

$$
\begin{aligned}
& \left\|\widetilde{\mathrm{d}}^{2 \varepsilon}\right\|_{\mathrm{H}^{1}(\Omega)} \leq C\left(\left\|\nabla \widetilde{u}^{0}-\nabla \widetilde{u}^{(0)}(0)-\mathcal{H}_{\widetilde{u}^{0}}(0) x\right\|_{\mathrm{L}^{2}\left(\Omega_{f}^{\varepsilon}\right)}+\varepsilon^{2}\|\nabla \widetilde{v}\|_{\mathrm{L}^{2}\left(\Omega_{f}^{\varepsilon}\right)}\right. \\
& \left.+\varepsilon^{2}\left\|\widetilde{W}^{1}\left(\frac{\cdot}{\varepsilon}\right)\right\|_{\mathrm{H}^{1 / 2}(\Gamma)}+\left\|-\varepsilon \mathcal{W}^{0}\left(\frac{\cdot}{\varepsilon}\right)-\varepsilon^{2} \frac{R^{0}}{r}\left(\widetilde{a}_{1} \cos (\theta)+\widetilde{b}_{1} \sin (\theta)\right)\right\|_{\mathrm{H}^{1 / 2}(\Gamma)}\right) .
\end{aligned}
$$

According to the expansion obtained in the previous section and definition of $\widetilde{v}$, it comes

$$
-\varepsilon \widetilde{W}^{0}\left(\frac{\dot{\varepsilon}}{\varepsilon}\right)-\varepsilon^{2} \frac{R^{0}}{r}\left(\widetilde{a}_{1} \cos (\theta)+\widetilde{b}_{1} \sin (\theta)\right)=-\sum_{n \geq 2}\left(\frac{R^{0}}{r}\right)^{n} \varepsilon^{n+1}\left(\widetilde{a}_{n} \cos (\theta)+\widetilde{b}_{n} \sin (\theta)\right) .
$$

Let $\Omega_{\mathrm{c}}$ regular subset of $\mathbb{R}^{2}$ such that $\Gamma \subset \Omega_{\mathrm{c}}$ and there exist $\left(r_{1}, r_{2}\right) \in \mathbb{R}^{+} \times \mathbb{R}^{+}$satisfying $r_{1}<r<r_{2}$ on $\Omega_{\mathrm{c}}$. Then we infer that

$$
\left\|\widetilde{W}^{0}\left(\begin{array}{c}
\cdot \\
\varepsilon
\end{array}\right)\right\|_{\mathrm{H}^{1 / 2}(\Gamma)} \leq C\left\|\widetilde{W}^{0}\left(\frac{\cdot}{\varepsilon}\right)\right\|_{\mathrm{H}^{1}\left(\Omega_{\mathrm{c}}\right)} \cdot
$$

Choosing $\varepsilon>0$ such that $\varepsilon<\frac{r_{1}}{2 R^{0}}$, we find

$$
\begin{aligned}
& \left\|-\nabla\left(\varepsilon \widetilde{W}^{0}\left(\frac{\cdot}{\varepsilon}\right)-\varepsilon^{2} \frac{R^{0}}{r}\left(\widetilde{a}_{1} \cos (\theta)+\widetilde{b}_{1} \sin (\theta)\right)\right)\right\|_{L^{2}\left(\Omega_{\mathrm{c}}\right)} \\
& \leq \frac{\left.4 \varepsilon R^{0} \sqrt{\mu\left(\Omega_{\mathrm{c}}\right.}\right)}{r_{1}^{2}} \sum_{n \geq 2}\left(n\left(\frac{R^{0}}{r_{1}}\right)^{n-1} \varepsilon^{n}\left(\widetilde{a}_{n} \cos (\theta)+\widetilde{b}_{n} \sin (\theta)\right)\right) \leq C \varepsilon^{3} .
\end{aligned}
$$


Furthermore, we have

$$
\begin{aligned}
& \left\|\varepsilon \widetilde{W}^{0}\left(\frac{\dot{\bar{\varepsilon}}}{\varepsilon}\right)-\varepsilon^{2} \frac{R^{0}}{r}\left(\widetilde{a}_{1} \cos (\theta)+\widetilde{b}_{1} \sin (\theta)\right)\right\|_{\mathrm{L}^{2}\left(\Omega_{\mathrm{c}}\right)} \\
& \leq \sum_{n \geq 2}\left(\left(\frac{R^{0}}{r_{1}}\right)^{n} \varepsilon^{n+1}\left(\widetilde{a}_{n} \cos (\theta)+\widetilde{b}_{n} \sin (\theta)\right)\right) \leq C \varepsilon^{3} .
\end{aligned}
$$

Clearly, it comes that

$$
\left\|\varepsilon \widetilde{W}^{0}\left(\frac{\cdot}{\varepsilon}\right)-\varepsilon^{2} \frac{R^{0}}{r}\left(\widetilde{a}_{1} \cos (\theta)+\widetilde{b}_{1} \sin (\theta)\right)\right\|_{L^{2}\left(\Omega_{\mathrm{c}}\right)} \leq C \varepsilon^{3}
$$

By using Cauchy-Schwarz's inequality, we immediately deduce that

$$
\left\|\nabla \widetilde{u}^{0}-\nabla \widetilde{u}^{(0)}(0)-\mathcal{H}_{\widetilde{u}^{0}}(0) x\right\|_{\mathrm{L}^{2}\left(\Omega_{f}^{\varepsilon}\right)} \leq C \varepsilon^{3},
$$

and since $\widetilde{v}$ is an harmonic function, its gradient is bounded, so that

$$
\|\nabla \widetilde{v}\|_{L^{2}\left(\Omega_{f}^{\varepsilon}\right)} \leq C \varepsilon
$$

As we already mentioned above, a similar approach to the one presented in the previous section for $\widetilde{W}^{0}$ is used to get $\widetilde{W}^{1}$. Namely, we have

$$
\left\|\widetilde{W}^{1}\left(\frac{\dot{\varepsilon}}{\varepsilon}\right)\right\|_{\mathrm{H}^{1 / 2}(\Gamma)} \leq C \varepsilon
$$

Clearly, we may deduce from the above estimates that

$$
\left\|\widetilde{d}^{2 \varepsilon}\right\|_{\mathrm{H}^{1}(\Omega)} \leq C \varepsilon^{3}
$$

\section{Thick-point approximation with small inclusion}

The present section is dedicated to approximate the displacement $u^{\varepsilon}$ in the inclusion $\Omega_{f}^{\varepsilon}$ in some finite dimensional space $Q^{\varepsilon}$ introduced later on. More precisely, we replace $\widetilde{u}^{\varepsilon}$ by $u^{\varepsilon}$ in (2.1) and we assume that $u_{f}^{\varepsilon}$ belongs to $\mathrm{Q}^{\varepsilon}$ in the inclusion $\Omega_{f}^{\varepsilon}$. Let us define the following spaces:

$\mathrm{V}_{\mathrm{U}}^{\varepsilon} \stackrel{\text { def }}{=}\left\{v \in \mathrm{H}^{1}(\Omega): v_{\left.\right|_{\Omega_{f}^{\varepsilon}}} \in \mathrm{Q}^{\varepsilon}\right.$ with $\left.v_{\left.\right|_{\Gamma}}=\mathrm{U}\right\}, \quad \mathrm{V}^{\varepsilon} \stackrel{\text { def }}{=}\left\{v \in \mathrm{H}^{1}(\Omega): v_{\left.\right|_{\Omega_{f}^{\varepsilon}}} \in \mathrm{Q}^{\varepsilon}\right\}, \quad \mathrm{V}_{0}^{\varepsilon} \stackrel{\text { def }}{=}\left\{v \in \mathrm{V}^{\varepsilon}: v_{\left.\right|_{\Gamma}}=0\right\}$.

Using Galerkin's principle, the weak formulation associated to the approximated problem is given by

$$
\left\{\begin{array}{l}
\text { find } u^{\varepsilon} \in \mathrm{V}^{\varepsilon} \text { such that for all } w \in \mathrm{V}_{0}^{\varepsilon} \\
\int_{\Omega_{f}^{\varepsilon}} \alpha_{f} \nabla u_{f}^{\varepsilon} \cdot \nabla w_{f} \mathrm{~d} x+\int_{\Omega_{m}^{\varepsilon}} \alpha_{m} \nabla u_{m}^{\varepsilon} \cdot \nabla w_{m} \mathrm{~d} x=0 .
\end{array}\right.
$$

The existence and uniqueness of a solution to problem (4.1) is rather classical and its verification is let to the reader. 


\subsection{A preliminary approximation result}

In order to write the asymptotic expansion of the approximate problem (4.1), let us introduce Proj $\mathrm{V}_{\mathrm{U}}^{\varepsilon}\left(\widetilde{u}^{0}\right)$ the orthogonal projection of $\widetilde{u}^{0}$ onto $\mathrm{V}_{\mathrm{U}}^{\varepsilon}$ in the following sense:

$$
\left\{\begin{array}{l}
\operatorname{Proj}_{\mathrm{V}_{\mathrm{U}}^{\varepsilon}}\left(\widetilde{u}^{0}\right) \in \mathrm{V}_{\mathrm{U}}^{\varepsilon} \text { and for all } w \in \mathrm{V}_{0}^{\varepsilon} \\
\int_{\Omega_{f}^{\varepsilon}} \nabla \operatorname{Proj}_{\mathrm{V}_{\mathrm{U}}^{\varepsilon}}\left(\widetilde{u}^{0}\right) \cdot \nabla w_{f} \mathrm{~d} x+\int_{\Omega_{m}^{\varepsilon}} \nabla \operatorname{Proj}_{\mathrm{V}_{\mathrm{U}}^{\varepsilon}}\left(\widetilde{u}^{0}\right) \cdot \nabla w_{m} \mathrm{~d} x=0 .
\end{array}\right.
$$

The following estimate between $\operatorname{Proj}_{\mathrm{V}_{\mathrm{U}}^{\varepsilon}}\left(\widetilde{u}^{0}\right)$ and $\widetilde{u}^{0}$ is intensively used later on.

Lemma 4.1. Assume that $\mathrm{Q}^{\varepsilon}$ contains $\mathrm{P}_{k}\left(\Omega_{f}^{\varepsilon}\right)$, the space of polynomials of degree less or equal to $k$ on $\Omega_{f}^{\varepsilon}$, then there exists a constant $C>0$ such that

$$
\left\|\operatorname{Proj}_{\mathrm{V}_{\mathrm{U}}^{\varepsilon}}\left(\widetilde{u}^{0}\right)-\widetilde{u}^{0}\right\|_{\mathrm{H}^{1}(\Omega)} \leq C \varepsilon^{k+1}
$$

Proof. Let $\varphi$ defined as the $k^{\text {th }}$ order rest of $\widetilde{u}^{0}$ Taylor expansion in $\Omega$. Let $K \subset \subset \Omega$ be a fixed compact neighborhood 0 . Since $\varphi$ is bounded in $\mathrm{C}^{\infty}(K)$, there exists a constant $C_{K}>0$, depending on $K$, such that

$$
\forall x \in K:|\varphi(x)| \leq C_{K}|x|^{k+1} \quad \text { and } \quad|\nabla \varphi(x)| \leq C_{K}|x|^{k} .
$$

In the sequel, we omit the index $K$ in the notation $C_{K}$. For all $\varepsilon>0$, we define $z^{\varepsilon} \in \mathrm{H}^{1}(\Omega)$ such that $z^{\varepsilon}=\widetilde{u}^{0}-\varphi$ on $\Omega_{f}^{\varepsilon}, z_{\left.\right|_{\Gamma} ^{\varepsilon}}^{\varepsilon}=\widetilde{u}^{0}-\varphi_{\left.\right|_{\Gamma} \varepsilon}, z_{\left.\right|_{\Gamma}}^{\varepsilon}=\mathrm{U}$ and

$$
\forall w \in \mathrm{H}_{0}^{1}\left(\Omega_{m}^{\varepsilon}\right): \int_{\Omega_{m}^{\varepsilon}} \nabla z^{\varepsilon} \cdot \nabla w \mathrm{~d} x=0
$$

Notice that

$$
\forall w \in \mathrm{H}_{0}^{1}\left(\Omega_{m}^{\varepsilon}\right): \int_{\Omega_{m}^{\varepsilon}} \nabla \widetilde{u}^{0} \cdot \nabla w \mathrm{~d} x=0
$$

which implies that

$$
\forall w \in \mathrm{H}_{0}^{1}\left(\Omega_{m}^{\varepsilon}\right): \int_{\Omega_{m}^{\varepsilon}} \nabla\left(z^{\varepsilon}-\widetilde{u}^{0}\right) \cdot \nabla w \mathrm{~d} x=0 .
$$

Let us define $\zeta \in \mathcal{D}\left(\mathbb{R}^{2} ; \mathbb{R}^{+}\right), 0 \leq \zeta \leq 1$, such that $\zeta=1$ and $\zeta=0$ in a neighborhood of $\bar{\Omega}_{f}^{1}$ and $\mathbb{R}^{2} \backslash \Omega$, respectively. For $\varepsilon>0$ and $x \in \mathbb{R}^{2}$, we define $\zeta^{\varepsilon} \stackrel{\text { def }}{=} \zeta(\dot{\bar{\varepsilon}})$ and $\psi^{\varepsilon} \stackrel{\text { def }}{=} \zeta^{\varepsilon} \varphi$. Thus it is clear that $\psi^{\varepsilon} \in \mathrm{H}^{1}\left(\Omega_{m}^{\varepsilon}\right)$ and $\psi_{\left.\right|_{\Gamma^{\varepsilon}} ^{\varepsilon}}^{\varepsilon}=\varphi_{\left.\right|_{\Gamma^{\varepsilon}}}$. We may infer that $\left(z^{\varepsilon}-\widetilde{u}^{0}+\psi^{\varepsilon}\right)_{\left.\right|_{\Gamma^{\varepsilon}}}=0$ and obviously $\left(z^{\varepsilon}-\widetilde{u}^{0}+\psi^{\varepsilon}\right)_{\left.\right|_{\Gamma}}=0$, which implies that $z^{\varepsilon}-\widetilde{u}^{0}+\psi^{\varepsilon} \in \mathrm{H}_{0}^{1}\left(\Omega_{m}^{\varepsilon}\right)$. On the other hand, identity (4.5) leads to

$$
\forall w \in \mathrm{H}_{0}^{1}\left(\Omega_{m}^{\varepsilon}\right): \int_{\Omega_{m}^{\varepsilon}} \nabla\left(z^{\varepsilon}-\widetilde{u}^{0}+\psi^{\varepsilon}\right) \cdot \nabla w \mathrm{~d} x=\int_{\Omega_{m}^{\varepsilon}} \nabla \psi^{\varepsilon} \cdot \nabla w \mathrm{~d} x
$$

Define

$$
\beta^{\varepsilon} \stackrel{\text { def }}{=} \begin{cases}z^{\varepsilon}-\widetilde{u}^{0}+\psi^{\varepsilon} & \text { on } \Omega_{m}^{\varepsilon} \\ 0 & \text { on } \Omega_{f}^{\varepsilon}\end{cases}
$$


Clearly, $\beta^{\varepsilon} \in \mathrm{H}_{0}^{1}(\Omega)$. Denote Poincaré's constant by $C_{\Omega}>0$, independent of $\varepsilon>0$, related to $\Omega$. Since $\beta^{\varepsilon}=0$ on $\Omega_{f}^{\varepsilon}$, we get

$$
\left\|\beta^{\varepsilon}\right\|_{\mathrm{H}^{1}(\Omega)} \leq C_{\Omega}\left\|\nabla \beta^{\varepsilon}\right\|_{\mathrm{L}^{2}(\Omega)} \leq C_{\Omega}\left\|\nabla \beta^{\varepsilon}\right\|_{\mathrm{L}^{2}\left(\Omega_{m}^{\varepsilon}\right)} .
$$

Taking $w=\beta_{\left.\right|_{\Omega_{m}^{\varepsilon}} ^{\varepsilon}}^{\varepsilon}$ into (4.6), and using Cauchy-Schwarz's inequality, we find

$$
\left\|\nabla \beta^{\varepsilon}\right\|_{L^{2}\left(\Omega_{m}^{\varepsilon}\right)} \leq C\left\|\nabla \psi^{\varepsilon}\right\|_{L^{2}\left(\Omega_{m}^{\varepsilon}\right)} .
$$

Hence, we have

$$
\left\|\beta^{\varepsilon}\right\|_{\mathrm{H}^{1}(\Omega)} \leq C\left\|\nabla \psi^{\varepsilon}\right\|_{\mathrm{L}^{2}\left(\Omega_{m}^{\varepsilon}\right)} .
$$

which implies that

$$
\left\|z^{\varepsilon}-\widetilde{u}^{0}+\psi^{\varepsilon}\right\|_{\mathrm{H}^{1}(\Omega)} \leq C\left\|\nabla \psi^{\varepsilon}\right\|_{\mathrm{L}^{2}\left(\Omega_{m}^{\varepsilon}\right)},
$$

and finally, according to the triangular inequality, we obtain

$$
\left\|z^{\varepsilon}-\widetilde{u}^{0}\right\|_{\mathrm{H}^{1}\left(\Omega_{m}^{\varepsilon}\right)} \leq C\left\|\psi^{\varepsilon}\right\|_{\mathrm{H}^{1}\left(\Omega_{m}^{\varepsilon}\right)} .
$$

It remains to estimate $\left\|\psi^{\varepsilon}\right\|_{\mathrm{H}^{1}\left(\Omega_{m}^{\varepsilon}\right)}$. To this aim, we first observe that

$$
\begin{aligned}
\int_{\Omega_{m}^{\varepsilon}}\left|\nabla \psi^{\varepsilon}\right|^{2} \mathrm{~d} x & \leq \int_{\Omega_{m}^{\varepsilon}}\left|\frac{1}{\varepsilon} \nabla \zeta\left(\frac{\cdot}{\varepsilon}\right) \varphi\right|^{2} \mathrm{~d} x+\int_{\Omega_{m}^{\varepsilon}}\left|\zeta\left(\frac{\dot{-}}{\varepsilon}\right) \nabla \varphi\right|^{2} \mathrm{~d} x \\
& \leq C\left(\frac{1}{\varepsilon^{2}} \int_{\operatorname{supp}\left(\zeta^{\varepsilon}\right)}|\varphi|^{2} \mathrm{~d} x+\int_{\operatorname{supp}\left(\zeta^{\varepsilon}\right)}|\nabla \varphi|^{2} \mathrm{~d} x\right)
\end{aligned}
$$

where $\operatorname{supp}\left(\zeta^{\varepsilon}\right)$ denotes the support of $\zeta^{\varepsilon}$. By using (4.4), we obtain

$$
\int_{\Omega_{m}^{\varepsilon}}\left|\nabla \psi^{\varepsilon}\right|^{2} \mathrm{~d} x \leq C \mu\left(\operatorname{supp}\left(\zeta^{\varepsilon}\right)\right) \varepsilon^{2 \mathrm{k}}
$$

where $\mu$ is Lebesgue's measure in $\mathbb{R}^{2}$. Since $\mu\left(\operatorname{supp}\left(\zeta^{\varepsilon}\right)\right) \leq \mathrm{C} \varepsilon^{2}$, it comes that

$$
\int_{\Omega_{m}^{\varepsilon}}\left|\nabla \psi^{\varepsilon}\right|^{2} \mathrm{~d} x \leq C \varepsilon^{2 k+2} .
$$

Once again by using (4.4), we find

$$
\left\|\psi^{\varepsilon}\right\|_{\mathrm{L}^{2}\left(\Omega_{m}^{\varepsilon}\right)}^{2}=\int_{\operatorname{supp}\left(\zeta^{\varepsilon}\right)}\left|\zeta^{\varepsilon} \varphi\right|^{2} \mathrm{~d} x \leq C \varepsilon^{2 k+2} \mu\left(\operatorname{supp}\left(\zeta^{\varepsilon}\right)\right) .
$$

It follows that

$$
\left\|\psi^{\varepsilon}\right\|_{\mathrm{L}^{2}\left(\Omega_{m}^{\varepsilon}\right)}^{2} \leq C \varepsilon^{2 k+4}
$$

which by using (4.6)-(4.8) leads to

$$
\left\|z^{\varepsilon}-\widetilde{u}^{0}\right\|_{\mathrm{H}^{1}\left(\Omega_{m}^{\varepsilon}\right)} \leq C \varepsilon^{k+1}
$$


Let us emphasize that

$$
\left\|z^{\varepsilon}-\widetilde{u}^{0}\right\|_{\mathrm{H}^{1}\left(\Omega_{f}^{\varepsilon}\right)}=\|\varphi\|_{\mathrm{H}^{1}\left(\Omega_{f}^{\varepsilon}\right)} \leq C \mu\left(\Omega_{f}^{\varepsilon}\right)\left(\varepsilon^{2 k+2}+\varepsilon^{2 k}\right) .
$$

Since the diameter of $\Omega_{f}^{\varepsilon}$ is smaller that $C \varepsilon$ and by using (4.4), we may infer that $\mu\left(\Omega_{f}^{\varepsilon}\right) \leq C \varepsilon^{2}$. Hence we get

$$
\left\|z^{\varepsilon}-\widetilde{u}^{0}\right\|_{\mathrm{H}^{1}\left(\Omega_{f}^{\varepsilon}\right)}^{2} \leq C \varepsilon^{2 k+2} .
$$

According to (4.9) and (4.10), we get

$$
\left\|z^{\varepsilon}-\widetilde{u}^{0}\right\|_{\mathrm{H}^{1}(\Omega)} \leq C \varepsilon^{k+1}
$$

Since $z^{\varepsilon} \in \mathrm{V}_{\mathrm{U}}^{\varepsilon}$, we may infer that

$$
\left\|\operatorname{Proj}_{\mathrm{V}_{\mathrm{U}}^{\varepsilon}}\left(\widetilde{u}^{0}\right)-\widetilde{u}^{0}\right\|_{\mathrm{H}^{1}\left(\Omega_{m}^{\varepsilon}\right)} \leq\left\|z^{\varepsilon}-\widetilde{u}^{0}\right\|_{\mathrm{H}^{1}\left(\Omega_{m}^{\varepsilon}\right)} \leq C \varepsilon^{k+1}
$$

which proves the lemma.

\subsection{First order asymptotic expansion for the approximated problem}

We establish now the first order asymptotic expansion for the approximated problem. Namely, we assume that $\mathrm{P}_{1}\left(\Omega_{f}^{\varepsilon}\right) \subset \mathrm{Q}^{\varepsilon}$ where $\mathrm{P}_{1}\left(\Omega_{f}^{\varepsilon}\right)$ denotes the space of polynomials of degree less or equal to 1 on $\Omega_{f}^{\varepsilon}$. From (4.2) and (4.1), we deduce that

$$
\begin{aligned}
& \int_{\Omega_{f}^{\varepsilon}} \alpha_{f} \nabla\left(u_{f}^{\varepsilon}-\operatorname{Proj}_{\mathrm{V}_{\mathrm{U}}^{\varepsilon}}\left(\widetilde{u}^{0}\right)\right) \cdot \nabla w_{f} \mathrm{~d} x+\int_{\Omega_{m}^{\varepsilon}} \alpha_{m} \nabla\left(u_{m}^{\varepsilon}-\operatorname{Proj}_{\mathrm{V}_{\mathrm{U}}^{\varepsilon}}\left(\widetilde{u}^{0}\right)\right) \cdot \nabla w_{m} \mathrm{~d} x \\
& +\int_{\Omega_{f}^{\varepsilon}}\left(\alpha_{f}-\alpha_{m}\right) \nabla \operatorname{Proj}_{\mathrm{V}_{\mathrm{U}}^{\varepsilon}}\left(\widetilde{u}^{0}\right) \cdot \nabla w_{f} \mathrm{~d} x=0
\end{aligned}
$$

for all $w \in \mathrm{V}_{0}^{\varepsilon}$. Then, we define $\mathrm{d}_{k}^{0 \varepsilon} \stackrel{\text { def }}{=} u_{k}^{\varepsilon}-\operatorname{Proj}_{\mathrm{V}_{\mathrm{U}}^{\varepsilon}}$ satisfying the following variational formulation:

$$
\left\{\begin{array}{l}
\mathrm{d}^{0 \varepsilon} \in \mathrm{V}_{0}^{\varepsilon} \text { and for all } w \in \mathrm{V}_{0}^{\varepsilon}, \\
\int_{\Omega_{f}^{\varepsilon}} \alpha_{f} \nabla \mathrm{d}^{0 \varepsilon} \cdot \nabla w_{f} \mathrm{~d} x+\int_{\Omega_{m}^{\varepsilon}} \alpha_{m} \nabla \mathrm{d}^{0 \varepsilon} \cdot \nabla w_{m} \mathrm{~d} x+\int_{\Omega_{f}^{\varepsilon}}\left(\alpha_{f}-\alpha_{m}\right) \nabla \operatorname{Proj}_{\mathrm{V}_{\mathrm{U}}^{\varepsilon}}\left(\widetilde{u}^{0}\right) \cdot \nabla w_{f} \mathrm{~d} x=0 .
\end{array}\right.
$$

It is also convenient to introduce the following functional spaces:

$$
\mathrm{V}_{\log } \stackrel{\text { def }}{=}\left\{v \in \widetilde{\mathrm{V}}_{\log }: v_{\mid \Omega^{1}} \in \mathrm{Q}^{1}\right\} \quad \text { and } \quad \mathrm{V}_{\log }^{R^{0}} \stackrel{\text { def }}{=}\left\{v \in \mathrm{V}_{\log }: \int_{-\pi}^{\pi} v\left(R^{0} \cos (\theta), R^{0} \sin (\theta)\right) \mathrm{d} \theta=0\right\} .
$$

Notice that the first order corrector $W^{0}$ for the $\mathrm{P}_{1}$ approximation is obtained by replacing $\operatorname{Proj}_{\mathrm{V}_{\mathrm{U}}^{\varepsilon}}\left(\widetilde{u}^{0}\right)$ in (4.12) by $\widetilde{u}^{0}$ and proceeding as in Section 3, we get

$$
\left\{\begin{array}{l}
W^{0} \in \mathrm{V}_{\log } \text { and for all } w \in \mathrm{V}_{\log }^{R^{0}}, \\
\int_{\Omega_{f}^{1}}\left(\alpha_{f}-\alpha_{m}\right) \nabla \widetilde{u}^{0}(0) \cdot \nabla w_{f} \mathrm{~d} y+\int_{\Omega_{f}^{1}} \alpha_{f} \nabla W_{f}^{0} \cdot \nabla w_{f} \mathrm{~d} y+\int_{\Omega^{\infty}} \alpha_{m} \nabla W_{m}^{0} \cdot \nabla w_{m} \mathrm{~d} y=0 .
\end{array}\right.
$$


Choosing $w$ such that $w \in \mathrm{V}_{0}^{\varepsilon}$ holds and its extension vanishes, it comes from (4.13) that

$$
\begin{aligned}
& \int_{\Omega_{f}^{\varepsilon}} \alpha_{f} \nabla\left(\varepsilon W_{f}^{0}\left(\frac{\cdot}{\varepsilon}\right)\right) \cdot \nabla w_{f} \mathrm{~d} x+\int_{\Omega_{m}^{\varepsilon}} \alpha_{m} \nabla\left(\varepsilon W_{m}^{0}\left(\frac{\dot{z}}{\varepsilon}\right)\right) \cdot \nabla w_{m} \mathrm{~d} x \\
& +\int_{\Omega_{f}^{\varepsilon}}\left(\alpha_{f}-\alpha_{m}\right) \nabla \widetilde{u}^{(0)}(0) \cdot \nabla w_{f} \mathrm{~d} x=0
\end{aligned}
$$

for all $w \in \mathrm{V}_{0}^{\varepsilon}$. Setting $\mathrm{d}^{1 \varepsilon} \stackrel{\text { def }}{=} u^{\varepsilon}-\operatorname{Proj}_{\mathrm{V}_{\mathrm{U}}^{\varepsilon}}\left(\widetilde{u}^{0}\right)-\varepsilon W^{0}(\dot{\bar{\varepsilon}})$, we have

$$
\left\{\begin{array}{l}
\mathrm{d}^{1 \varepsilon} \in \mathrm{V}^{\varepsilon} \text { and for all } w \in \mathrm{V}_{0}^{\varepsilon} \\
\int_{\Omega_{f}^{\varepsilon}} \alpha_{f} \nabla \mathrm{d}^{1 \varepsilon} \cdot \nabla w_{f} \mathrm{~d} x+\int_{\Omega_{m}^{\varepsilon}} \alpha_{m} \nabla \mathrm{d}^{1 \varepsilon} \cdot \nabla w_{m} \mathrm{~d} x \\
+\int_{\Omega_{f}^{\varepsilon}}\left(\alpha_{f}-\alpha_{m}\right)\left(\nabla \widetilde{u}^{(0)}(0)-\nabla \operatorname{Proj}_{\mathrm{V}_{\mathrm{U}}^{\varepsilon}}\left(\widetilde{u}^{0}\right)\right) \cdot \nabla w_{f} \mathrm{~d} x=0 \\
\text { with } \mathrm{d}^{1 \varepsilon}=-\varepsilon W^{0}\left(\frac{\cdot}{\varepsilon}\right) \text { on } \Gamma
\end{array}\right.
$$

Lemma 4.2. There exists a constant $C>0$, independent of $\varepsilon$, such that

$$
\left\|\mathrm{d}^{1 \varepsilon}\right\|_{\mathrm{H}^{1}(\Omega)} \leq C \varepsilon^{2}
$$

Proof. The strategy adopted here is quite similar to the one developed in the proof of Lemma 3.1. First, we may observe that

$$
\left\|\mathrm{d}^{1 \varepsilon}\right\|_{\mathrm{H}^{1}(\Omega)} \leq C\left(\left\|\nabla \widetilde{u}^{(0)}(0)-\nabla \operatorname{Proj}_{\mathrm{V}_{\mathrm{U}}^{\varepsilon}}\left(\widetilde{u}^{0}\right)\right\|_{\mathrm{L}^{2}\left(\Omega_{f}^{\varepsilon}\right)}+\varepsilon\left\|W^{0}(\dot{\bar{\varepsilon}})\right\|_{\mathrm{H}^{1 / 2}(\Gamma)}\right)
$$

Arguing as for $\widetilde{W}^{0}$ introduced in Section $3, W^{0}$ is an harmonic function on $\Omega^{\infty}$ and satisfies the following identity:

$$
W^{0}\left(\frac{x}{\varepsilon}\right)=\sum_{n \geq 1}\left(\frac{\varepsilon R^{0}}{r}\right)^{n}\left(a_{n} \cos (n \theta)+b_{n} \sin (n \theta)\right) .
$$

Therefore, we have

$$
\left\|\mathrm{d}^{1 \varepsilon}\right\|_{\mathrm{H}^{1}(\Omega)} \leq C\left(\left\|\nabla \widetilde{u}^{(0)}(0)-\nabla \widetilde{u}^{(0)}\right\|_{\mathrm{L}^{2}\left(\Omega_{f}^{\varepsilon}\right)}+\left\|\nabla\left(\widetilde{u}^{(0)}-\operatorname{Proj}_{\mathrm{V}_{\mathrm{U}}^{\varepsilon}}\left(\widetilde{u}^{0}\right)\right)\right\|_{\mathrm{L}^{2}\left(\Omega_{f}^{\varepsilon}\right)}\right)+C \varepsilon^{2}
$$

and we may conclude the proof

\subsection{Second order asymptotic expansion for the approximated problem}

We exhibit the second order asymptotic expansion for the approximated problem. Namely, we assume that $\mathrm{P}_{2}\left(\Omega_{f}^{\varepsilon}\right) \subset \mathrm{Q}^{\varepsilon}$ where $\mathrm{P}_{2}\left(\Omega_{f}^{\varepsilon}\right)$ denotes the space of polynomials of degree less or equal to 2 on $\Omega_{f}^{\varepsilon}$. Let us define the following spaces:

$$
\mathrm{V}_{v} \stackrel{\text { def }}{=}\left\{v \in \mathrm{H}^{1}(\Omega): v_{\left.\right|_{\Gamma}}=-\frac{R^{0}}{r}\left(a_{1} \cos (\theta)+b_{1} \cos (\theta)\right)\right\} \quad \text { and } \quad \mathrm{V}_{v}^{\varepsilon} \stackrel{\text { def }}{=}\left\{v \in \mathrm{V}_{v}: v_{\left.\right|_{\Omega_{f}^{\varepsilon}}} \in \mathrm{Q}^{\varepsilon}\right\} .
$$


On the one hand, let $v$ be the solution to the following problem:

$$
\left\{\begin{array}{l}
\text { Find } v \in \mathrm{V}_{v} \text { such that for all } w \in \mathrm{H}_{0}^{1}(\Omega), \\
\int_{\Omega} \alpha_{m} \nabla v \cdot \nabla w \mathrm{~d} x=0
\end{array}\right.
$$

On the other hand, let $W^{1}$ be a corrector defined by a scaled form of (4.13) in a dilated geometry satisfying

$$
\left\{\begin{array}{l}
W^{1} \in \mathrm{V}_{\log }^{R^{0}} \text { and for all } w \in \mathrm{V}_{\mathrm{log}}, \\
\int_{\Omega_{f}^{1}}\left(\alpha_{f}-\alpha_{m}\right) \mathcal{H}_{\widetilde{u}^{0}}(0) y \cdot \nabla w_{f} \mathrm{~d} y+\int_{\Omega_{f}^{1}} \alpha_{f} \nabla W_{f}^{1} \cdot \nabla w_{f} \mathrm{~d} y+\int_{\Omega^{\infty}} \alpha_{m} \nabla W_{m}^{1} \cdot \nabla w_{m} \mathrm{~d} y=0 .
\end{array}\right.
$$

Choosing $w \in \mathrm{V}_{0}^{\varepsilon}$, we get

$$
\begin{aligned}
& \int_{\Omega_{f}^{\varepsilon}} \alpha_{f} \nabla\left(\varepsilon^{2} W_{f}^{1}\left(\frac{\cdot}{\varepsilon}\right)\right) \cdot \nabla w_{f} \mathrm{~d} x+\int_{\Omega_{m}^{\varepsilon}} \alpha_{m} \nabla\left(\varepsilon^{2} W_{m}^{1}\left(\frac{\cdot}{\varepsilon}\right)\right) \cdot \nabla w_{m} \mathrm{~d} x \\
& +\int_{\Omega_{f}^{\varepsilon}}\left(\alpha_{f}-\alpha_{m}\right) \mathcal{H}_{\widetilde{u}^{0}}(0) x \cdot \nabla w_{f} \mathrm{~d} x=0 .
\end{aligned}
$$

for all $w \in \mathrm{V}_{0}^{\varepsilon}$. Setting $\mathrm{d}^{2 \varepsilon}=u^{\varepsilon}-\operatorname{Proj}_{\mathrm{V}_{\mathrm{U}}^{\varepsilon}}\left(\widetilde{u}^{0}\right)-\varepsilon W^{0}(\dot{\bar{\varepsilon}})-\varepsilon^{2} W^{1}(\dot{\bar{\varepsilon}})-\varepsilon^{2} \operatorname{Proj}_{\mathrm{V}_{v}^{\varepsilon}}(v)$. We may deduce from (3.16), (4.22), (4.12) and (4.1) that

$$
\left\{\begin{array}{l}
\mathrm{d}^{2 \varepsilon} \in \mathrm{V}_{\mathrm{U}}^{\varepsilon} \text { and for all } w \in \mathrm{V}_{\varepsilon}^{0} \\
\int_{\Omega_{f}^{\varepsilon}}\left(\alpha_{f}-\alpha_{m}\right)\left(\nabla \operatorname{Proj}_{\mathrm{V}_{\mathrm{U}}^{\varepsilon}} \widetilde{u}^{0}-\nabla \widetilde{u}^{(0)}(0)-\mathcal{H}_{\widetilde{u}^{0}}(0) x-\varepsilon^{2} \nabla v\right) \cdot \nabla w_{f} \mathrm{~d} x \\
+\int_{\Omega_{f}^{\varepsilon}} \alpha_{f} \nabla \mathrm{d}^{2 \varepsilon} \cdot \nabla w_{f} \mathrm{~d} x+\int_{\Omega_{m}^{\varepsilon}} \alpha_{m} \nabla \mathrm{d}^{2 \varepsilon} \cdot \nabla w_{m} \mathrm{~d} x=0 \\
\text { with } \mathrm{d}^{2 \varepsilon}=-\varepsilon W^{0}\left(\frac{\dot{-}}{\varepsilon}\right)-\varepsilon^{2} W^{1}\left(\frac{\cdot}{\varepsilon}\right)-\varepsilon^{2} \operatorname{Proj}_{\mathrm{V}_{\mathrm{U}}^{\varepsilon}}(v) \text { on } \Gamma .
\end{array}\right.
$$

Now proceeding as in Section 3.2, we may easily deduce the following lemma:

Lemma 4.3. There exists a constant $C>0$, independent of $\varepsilon$, such that

$$
\left\|\mathrm{d}^{2 \varepsilon}\right\|_{\mathrm{H}^{1}(\Omega)} \leq C \varepsilon^{3} .
$$

\section{Error estimate for the thick-point approximation}

The asymptotic expansion of the two preceeding sections allow us to give some estimates on the thickpoint approximation order. We treat several cases depending on the choice of the finite dimensional space $\mathrm{Q}^{\varepsilon}$. We begin with the polynomial spaces $\mathrm{P}_{1}\left(\Omega_{f}^{\varepsilon}\right)$ and $\mathrm{P}_{2}\left(\Omega_{f}^{\varepsilon}\right)$. Then, we enrich these two spaces with a few functions which span the correctors $\widetilde{W}^{0}$ solution to (3.6) and $\widetilde{W}^{1}$ solution to (3.15). More precisely, let

$$
\widetilde{W}^{0,0} \text { and } \widetilde{W}^{0,1} \text {, }
$$


be the solutions to $(3.6)$ for $\nabla \widetilde{u}^{0}(0)=\left(\begin{array}{l}1 \\ 0\end{array}\right)$ and $\nabla \widetilde{u}^{0}(0)=\left(\begin{array}{l}0 \\ 1\end{array}\right)$, respectively, and consider

$$
\mathrm{Q}_{1}^{\varepsilon} \stackrel{\text { def }}{=} \operatorname{Span}\left\{\mathrm{P}_{1}\left(\Omega_{f}^{\varepsilon}\right),\left.\widetilde{W}^{0,0}\left(\frac{\cdot}{\varepsilon}\right)\right|_{\Omega_{f}^{\varepsilon}},\left.\widetilde{W}^{0,1}\left(\frac{\cdot}{\varepsilon}\right)\right|_{\Omega_{f}^{\varepsilon}}\right\}
$$

Similarly, noting that $\operatorname{Trace}\left(\mathcal{H}_{\widetilde{u}^{0}}(0)\right)=0$ since $\widetilde{u}^{0}$ is a harmonic function, let also

$$
\widetilde{W}^{1,0} \text { and } \widetilde{W^{1,1}}
$$

be the solutions to $(3.15)$ for $\mathcal{H}_{\widetilde{u}^{0}}(0)=\left(\begin{array}{cc}1 & 0 \\ 0 & -1\end{array}\right)$, and $\mathcal{H}_{\widetilde{u}^{0}}(0)=\left(\begin{array}{ll}0 & 1 \\ 1 & 0\end{array}\right)$, respectively, and consider

$$
\mathrm{Q}_{2}^{\varepsilon}=\operatorname{Span}\left\{\mathrm{P}_{2}\left(\Omega_{f}^{\varepsilon}\right),\left.\widetilde{W}^{0,0}\left(\frac{\cdot}{\varepsilon}\right)\right|_{\Omega_{f}^{\varepsilon}},\left.\widetilde{W}^{0,1}\left(\frac{\cdot}{\varepsilon}\right)\right|_{\Omega_{f}^{\varepsilon}}, \widetilde{W}^{1,0}\left(\frac{\cdot}{\varepsilon}\right)_{\Omega_{f}^{\varepsilon}},\left.\widetilde{W}^{1,1}\left(\frac{\cdot}{\varepsilon}\right)\right|_{\Omega_{f}^{\varepsilon}}\right\} .
$$

Obviously, the restriction on $\Omega_{f}^{\varepsilon}$ of any $\widetilde{W}^{0}(\dot{\bar{\varepsilon}})$ solution to (3.6) belong to $\mathrm{Q}_{1}^{\varepsilon}$ and $\mathrm{Q}_{2}^{\varepsilon}$ and the restriction on $\Omega_{f}^{\varepsilon}$ of any $\widetilde{W}^{1}\left(\frac{\dot{\varepsilon}}{\varepsilon}\right)$ solution to (3.15) into $\mathrm{Q}_{2}^{\varepsilon}$. We introduce functionals $|\cdot|_{1, \Omega}$ on $\mathrm{L}^{2}(\Omega)$ as follows:

$$
|u|_{1, \Omega} \stackrel{\text { def }}{=}\|\nabla u\|_{L^{2}(\Omega)} .
$$

When using only $\mathrm{P}^{1}\left(\Omega_{f}^{\varepsilon}\right)$, we can set the following result.

Lemma 5.1. If $\mathrm{P}^{1}\left(\Omega_{f}^{\varepsilon}\right) \subset \mathrm{Q}^{\varepsilon}$, then there exists a constant $C>0$ such that:

$$
\left\|\widetilde{u}^{\varepsilon}-u^{\varepsilon}\right\|_{H^{1}(\Omega)} \leq C \varepsilon .
$$

Proof. Observe first that

$$
\widetilde{u}^{\varepsilon}-u^{\varepsilon}=\widetilde{u}^{0}+\varepsilon \widetilde{W}^{0}\left(\frac{\cdot}{\varepsilon}\right)+\widetilde{\mathrm{d}}^{1 \varepsilon}-\operatorname{Proj}_{\mathrm{V}_{\mathrm{U}}^{\varepsilon}}\left(\widetilde{u}^{0}\right)+\varepsilon W^{0}\left(\frac{\cdot}{\varepsilon}\right)+\mathrm{d}^{1 \varepsilon} .
$$

Since both $\left\|\widetilde{\mathrm{d}}^{1 \varepsilon}\right\|_{\mathrm{H}^{1}(\Omega)}$ and $\left\|\mathrm{d}^{1 \varepsilon}\right\|_{\mathrm{H}^{1}(\Omega)}$ are $\mathcal{O}\left(\varepsilon^{2}\right)$, using the fact that $\widetilde{u}^{\varepsilon}-u^{\varepsilon} \in \mathrm{H}_{0}^{1}(\Omega)$ and the equivalence of the norm and semi-norm in $\mathrm{H}_{0}^{1}(\Omega)$, we obtain

$$
\left\|\widetilde{u}^{\varepsilon}-u^{\varepsilon}\right\|_{\mathrm{H}^{1}(\Omega)} \leq C\left(\left\|\widetilde{u}^{0}-\operatorname{Proj}_{\mathrm{V}_{\mathrm{U}}^{\varepsilon}}\left(\widetilde{u}^{0}\right)\right\|_{\mathrm{H}^{1}(\Omega)}+\varepsilon\left|\widetilde{W}^{0}\left(\frac{\cdot}{\varepsilon}\right)-W^{0}\left(\frac{\cdot}{\varepsilon}\right)\right|_{1, \Omega}+\varepsilon^{2}\right) .
$$

The difference $\left\|\widetilde{u}^{0}-\operatorname{Proj}_{\mathrm{V}_{\mathrm{U}}^{\varepsilon}}\left(\widetilde{u}^{0}\right)\right\|_{\mathrm{H}^{1}(\Omega)}$ is $\mathcal{O}\left(\varepsilon^{2}\right)$ according to Lemma 4.1 and since $\mathrm{P}_{1}\left(\Omega_{f}^{\varepsilon}\right) \subset \mathrm{Q}^{\varepsilon}$. Finally, using $\widetilde{W}^{0} \in \mathrm{V}_{\log }^{R^{0}}$ and $\widetilde{W}^{0} \in \widetilde{\mathrm{V}}_{\log }^{R^{0}}$ the evaluation of $\left|\widetilde{W}^{0}\left(\frac{\dot{\varepsilon}}{\varepsilon}\right)-W^{0}\left(\frac{\dot{\varepsilon}}{\varepsilon}\right)\right|_{1, \Omega}$ leads to

$$
\left|\widetilde{W}^{0}\left(\begin{array}{c}
\cdot \\
\varepsilon
\end{array}\right)-W^{0}\left(\frac{\cdot}{\varepsilon}\right)\right|_{1, \Omega}^{2} \leq \int_{\frac{\Omega}{\varepsilon}}\left|\frac{1}{\varepsilon} \varepsilon \nabla\left(\widetilde{W}^{0}-W^{0}\right)\right|^{2} \frac{1}{\varepsilon} \mathrm{d} y=\int_{\mathbb{R}^{2}}\left|\nabla\left(\widetilde{W}^{0}-W^{0}\right)\right|^{2} \mathrm{~d} y \leq C,
$$

which ends the proof. 
Clearly, the fact that the term $\left|\widetilde{W}^{0}\left(\frac{\cdot}{\varepsilon}\right)-W^{0}\left(\frac{\dot{\varepsilon}}{\varepsilon}\right)\right|_{1, \Omega}$ is limited to being $\mathcal{O}(\varepsilon)$ is a source of nonoptimality. Furthermore, this estimate is independent of both $\varepsilon$ and $\mathrm{Q}^{\varepsilon}$. This indicates that the best possible estimate when $\mathrm{Q}^{\varepsilon}=\mathrm{P}^{2}\left(\Omega_{f}^{\varepsilon}\right)$ is not better than $\left\|\widetilde{u}^{\varepsilon}-u^{\varepsilon}\right\|_{\mathrm{H}^{1}(\Omega)}=\mathcal{O}(\varepsilon)$. We can improve this by using $\mathrm{Q}_{1}^{\varepsilon}$ and $\mathrm{Q}_{2}^{\varepsilon}$ as follows.

Lemma 5.2. If $\mathrm{Q}_{1}^{\varepsilon} \subset \mathrm{Q}^{\varepsilon}$ then there exists $C>0$ such that:

$$
\left\|\widetilde{u}^{\varepsilon}-u^{\varepsilon}\right\|_{\mathrm{H}^{1}(\Omega)} \leq C \varepsilon^{2}
$$

Proof. Clearly, when $\mathrm{Q}_{1}^{\varepsilon} \subset \mathrm{Q}^{\varepsilon}$, we have $\widetilde{W}^{0} \in \mathrm{V}_{\log }^{R^{0}}$, so that $\widetilde{W}^{0}=W^{0}$. Consequently, the term $\left|\widetilde{W}^{0}\left(\frac{\dot{\varepsilon}}{\varepsilon}\right)-W^{0}\left(\frac{\dot{\varepsilon}}{\varepsilon}\right)\right|_{1, \Omega}$ is vanishing which gives the result, following the proof of Lemma 5.1.

Lemma 5.3. If $\mathrm{Q}_{2}^{\varepsilon} \subset \mathrm{Q}^{\varepsilon}$ then there exists $C>0$ such that:

$$
\left\|\widetilde{u}^{\varepsilon}-u^{\varepsilon}\right\|_{\mathrm{H}^{1}(\Omega)} \leq C \varepsilon^{3} .
$$

Proof. Using the order two expansion, we have

$$
\begin{aligned}
\widetilde{u}^{\varepsilon}-u^{\varepsilon}= & \widetilde{u}^{0}+\varepsilon \widetilde{W}^{0}\left(\frac{\dot{-}}{\varepsilon}\right)+\varepsilon^{2} \widetilde{W}^{1}\left(\frac{\dot{\varepsilon}}{\varepsilon}\right)+\varepsilon^{2} \widetilde{v}+\widetilde{\mathrm{d}}^{2 \varepsilon} \\
& -\operatorname{Proj}_{\mathrm{V}_{\mathrm{U}}^{\varepsilon}}\left(\widetilde{u}^{0}\right)-\varepsilon W^{0}\left(\frac{\dot{\bar{\varepsilon}}}{\varepsilon}\right)-\varepsilon^{2} W^{1}\left(\frac{\cdot}{\varepsilon}\right)-\varepsilon^{2}\left(\operatorname{Proj}_{\mathrm{V}_{v}^{\varepsilon}} v\right)-\mathrm{d}^{2 \varepsilon} .
\end{aligned}
$$

We established that both $\left\|\widetilde{\mathrm{d}}^{2 \varepsilon}\right\|_{\mathrm{H}^{1}(\Omega)}$ and $\left\|\mathrm{d}^{2 \varepsilon}\right\|_{\mathrm{H}^{1}(\Omega)}$ are $\mathcal{O}\left(\varepsilon^{3}\right)$. Now, since $\mathrm{Q}_{2}^{\varepsilon} \subset \mathrm{Q}^{\varepsilon}$, both $\widetilde{W}^{0}$ and $\widetilde{W}^{1}$ belong to $\mathrm{V}_{\log }^{R^{0}}$, so that $\widetilde{W^{0}}=W^{0}$ and $\widetilde{W}^{1}=W^{1}$. Moreover, $\widetilde{W}^{0}=W^{0}$ implies also $v=\widetilde{v}$. Consequently, we get

$$
\left\|\widetilde{u}^{\varepsilon}-u^{\varepsilon}\right\|_{\mathrm{H}^{1}(\Omega)} \leq C\left(\left\|\widetilde{u}^{0}-\operatorname{Proj}_{\mathrm{V}_{\mathrm{U}}^{\varepsilon}}\left(\widetilde{u}^{0}\right)\right\|_{\mathrm{H}^{1}(\Omega)}+\left\|\widetilde{v}-\operatorname{Proj}_{\mathrm{V}_{v}^{\varepsilon}}(\widetilde{v})\right\|_{H^{1}(\Omega)}+\epsilon^{3}\right)
$$

Using Lemma 4.1 for both $\widetilde{u}^{0}$ and $\widetilde{v}$ and since $\mathrm{P}_{2}\left(\Omega_{f}^{\varepsilon}\right) \subset \mathrm{Q}^{\varepsilon}$ we deduce the desired result.

It is also possible to recover an optimal estimate using a norm on a crown-shaped domain excluding a small zone around the inclusion. For an arbitrary fixed $r>0$, we establish the following result.

Lemma 5.4. For $r>0$ small enough and $\mathrm{P}^{1}\left(\Omega_{f}^{\varepsilon}\right) \subset \mathrm{Q}^{\varepsilon}$, there exist a constant $C>0$ such that

$$
\left\|\widetilde{u}^{\varepsilon}-u^{\varepsilon}\right\|_{\mathrm{H}^{1}(\Omega \backslash \mathbb{B}(O, r))} \leq C \varepsilon^{2} .
$$

Proof. Following the proof of Lemma 5.1, we still obtain

$$
\left\|\widetilde{u}^{\varepsilon}-u^{\varepsilon}\right\|_{H^{1}(\Omega \backslash \mathbb{B}(O, r))} \leq C\left(\varepsilon\left|\widetilde{W}^{0}\left(\frac{\cdot}{\varepsilon}\right)-W^{0}\left(\frac{\dot{\varepsilon}}{\varepsilon}\right)\right|_{1, \Omega \backslash \mathbb{B}(O, r)}+\varepsilon^{2}\right) .
$$

We conclude by using the expansions of $\widetilde{W}^{0}$ and $W^{0}$ given by (3.10) and (4.17), respectively, and by obtaining

$$
\left|\widetilde{W}^{0}\left(\frac{\dot{\varepsilon}}{\varepsilon}\right)-W^{0}\left(\frac{\dot{c}}{\varepsilon}\right)\right|_{1, \Omega \backslash \mathbb{B}(O, r)}^{2}=\int_{\Omega \backslash \mathbb{B}(O, r)}\left|\nabla\left(\widetilde{W}^{0}\left(\frac{\dot{c}}{\varepsilon}\right)-W^{0}\left(\frac{\dot{\varepsilon}}{\varepsilon}\right)\right)\right|^{2} \mathrm{~d} x \leq C \varepsilon^{2}
$$




\section{Special case of the circular inclusion}

If we consider the special case of a circular inclusion, it is possible to give the explicit expression of the first and second order correctors $\widetilde{W}^{0}$ and $\widetilde{W}^{1}$. Indeed, from the weak form (3.6) and (3.15), integrating by part and using the fact that $\operatorname{Trace}\left(\mathcal{H}_{\widetilde{u}^{0}}(0)\right)=0$ since $\widetilde{u}^{0}$ is a harmonic function, they are solution to the following strong problem for $i \in\{0,1\}$ :

$$
\begin{aligned}
&-\alpha_{f} \Delta \widetilde{W}_{f}^{i}=0 \text { in } \Omega_{f}^{1}, \\
&-\alpha_{m} \Delta \widetilde{W}_{m}^{i}=0 \text { in } \Omega^{\infty}, \\
& \widetilde{W}_{f}^{i}=\widetilde{W}_{m}^{i} \text { on } \Gamma^{1}, \\
& \alpha_{f} \partial_{\mathbf{n}} \widetilde{W}_{f}^{i}=\alpha_{m} \partial_{\mathbf{n}} \widetilde{W}_{m}^{i}+\phi^{i} \text { on } \Gamma^{1}, \\
& \int_{-\pi}^{\pi} \widetilde{W}_{m}^{i}\left(R^{0} \cos (\theta), R^{0} \sin (\theta)\right) d \theta=0,
\end{aligned}
$$

with $\phi^{0} \stackrel{\text { def }}{=} \nabla \widetilde{u}^{0}(0) \cdot\left(\begin{array}{l}\cos (\theta) \\ \sin (\theta)\end{array}\right)$ and $\phi^{1} \stackrel{\text { def }}{=}\left(\mathcal{H}_{\widetilde{u}^{0}}(0)\left(\begin{array}{c}\cos (\theta) \\ \sin (\theta)\end{array}\right)\right) \cdot\left(\begin{array}{c}\cos (\theta) \\ \sin (\theta)\end{array}\right)$. The domain $\Omega_{f}^{1}$ being the unit disk in that case, and taking into account $(6.1 \mathrm{e})$ and the continuity condition (6.1c), we can expand $\widetilde{W}^{0}$ and $\widetilde{W}^{1}$ as follows for $i \in\{0,1\}$ :

$$
\begin{aligned}
& \widetilde{W}_{f}^{i}(y)=\sum_{n=1}^{+\infty} r^{n}\left(\widetilde{a}^{i, n} \cos (n \theta)+\widetilde{b}^{i, n} \sin (n \theta)\right), \\
& \widetilde{W}_{m}^{i}(y)=\sum_{n=1}^{+\infty} r^{-n}\left(\widetilde{a}^{i, n} \cos (n \theta)+\widetilde{b}^{i, n} \sin (n \theta)\right) .
\end{aligned}
$$

Concerning $\widetilde{W^{0}}$ and denoting $\kappa \stackrel{\text { def }}{=} \frac{\alpha_{m}-\alpha_{f}}{\alpha_{m}+\alpha_{f}}$, the condition (6.1d) implies

$$
\left(\begin{array}{l}
\widetilde{a}^{0,1} \\
\widetilde{b}^{0,1}
\end{array}\right)=\kappa \nabla \widetilde{u}^{0}(0), \quad \widetilde{a}^{0, n}=\widetilde{b}^{0, n}=0, \quad \text { for } n \geq 2,
$$

and denoting $\mathcal{H}_{\widetilde{u}^{0}}(0)=\left(\begin{array}{ll}h_{11} & h_{12} \\ h_{12} & h_{22}\end{array}\right)$, it implies for $\widetilde{W}^{1}$ :

$$
\widetilde{a}^{1,2}=\frac{\kappa}{2}\left(h_{11}-h_{22}\right), \quad \widetilde{b}^{1,2}=\kappa h_{12}, \quad \widetilde{a}^{0, n}=\widetilde{b}^{0, n}=0, \quad \text { for } n \neq 2 .
$$

This leads to the following expression of $\widetilde{W^{0}}$ and $\widetilde{W^{1}}$ :

$$
\begin{aligned}
& \widetilde{W}_{f}^{0}(y)=\kappa \nabla \widetilde{u}^{0}(0) \cdot y, \quad \widetilde{W}_{m}^{0}(y)=\frac{1}{|y|^{2}} \kappa \nabla \widetilde{u}^{0}(0) \cdot y \\
& \widetilde{W}_{f}^{1}(y)=\kappa\left(\left(h_{11}-h_{22}\right)\left(y_{1}^{2}-y_{2}^{2}\right)+2 h_{12} y_{1} y_{2}\right), \quad \widetilde{W}_{f}^{1}(y)=\frac{1}{|y|^{2}} \kappa\left(\left(h_{11}-h_{22}\right)\left(y_{1}^{2}-y_{2}^{2}\right)+2 h_{12} y_{1} y_{2}\right) .
\end{aligned}
$$

It is to be noted that $\widetilde{W}_{f}^{0}\left(\frac{\dot{\varepsilon}}{\varepsilon}\right) \in \mathrm{P}_{1}\left(\Omega_{f}^{\varepsilon}\right)$ and $\widetilde{W}_{f}^{1}\left(\frac{\dot{\varepsilon}}{\varepsilon}\right) \in \mathrm{P}_{2}\left(\Omega_{f}^{\varepsilon}\right)$. This means that, with reference to the notations of Section $5, \mathrm{Q}_{1}^{\varepsilon}=\mathrm{P}_{1}\left(\Omega_{f}^{\varepsilon}\right)$ and $\mathrm{Q}_{2}^{\varepsilon}=\mathrm{P}_{2}\left(\Omega_{f}^{\varepsilon}\right)$, which, thanks to Lemmas 5.2 and 5.3, leads to the following result, meaning that the optimality is reached: 
Lemma 6.1. In the case of a circular inclusion, if $\mathrm{P}_{1}\left(\Omega_{f}^{\varepsilon}\right) \subset \mathrm{Q}^{\varepsilon}$, then there exists $C>0$ such that

$$
\left\|\widetilde{u}^{\varepsilon}-u^{\varepsilon}\right\|_{\mathrm{H}^{1}(\Omega)} \leq C \varepsilon^{2}
$$

and if $\mathrm{P}_{2}\left(\Omega_{f}^{\varepsilon}\right) \subset \mathrm{Q}^{\varepsilon}$, then there exists $C>0$ such that

$$
\left\|\widetilde{u}^{\varepsilon}-u^{\varepsilon}\right\|_{\mathrm{H}^{1}(\Omega)} \leq C \varepsilon^{3}
$$

\section{Strong form of the thick-point approximation in the case $\mathrm{Q}^{\varepsilon}=\mathrm{P}_{1}\left(\Omega_{f}^{\varepsilon}\right)$}

We introduced the thick-point approximation in Section 4 in a weak form only. It is of course possible to deduce a strong form of the correpsonding problem. The aim of this section is to give the strong form when $\mathrm{Q}^{\varepsilon}=\mathrm{P}_{1}\left(\Omega_{f}^{\varepsilon}\right)$. Since in that case $u_{f}^{\varepsilon}$ belongs to $\mathrm{P}_{1}\left(\Omega_{f}^{\varepsilon}\right)$, it comes that there exists $\left(a_{f}^{\varepsilon}, b_{f}^{\varepsilon}, c_{f}^{\varepsilon}\right) \in \mathbb{R}^{3}$ such that

$$
u_{f}^{\varepsilon}\left(x_{1}, x_{2}\right)=a_{f}^{\varepsilon} x_{1}+b_{f}^{\varepsilon} x_{2}+c_{f}^{\varepsilon} .
$$

According to Green's formulae, we may deduce from (4.1) that for all $w \in \mathrm{V}_{\mathrm{U}}^{\varepsilon}$, we have

$$
\alpha_{f} \nabla u_{f}^{\varepsilon} \cdot \nabla w_{f} \mu\left(\Omega_{f}^{\varepsilon}\right)+\int_{\Omega_{m}^{\varepsilon}} \alpha_{m} \Delta u_{m}^{\varepsilon} w_{m} \mathrm{~d} x+\int_{\Gamma^{\varepsilon}} \alpha_{m} \partial_{\mathbf{n}} u_{m}^{\varepsilon} w_{m} \mathrm{~d} s=0 .
$$

Since $w \in \mathrm{V}_{0}^{\varepsilon}$, we may infer that $w_{f}=w_{m}$ and (7.1) can be rewritten as follows:

$$
\alpha_{f} \nabla u_{f}^{\varepsilon} \cdot \nabla w_{f} \mu\left(\Omega_{f}^{\varepsilon}\right)+\int_{\Omega_{m}^{\varepsilon}} \alpha_{m} \Delta u_{m}^{\varepsilon} w_{m} \mathrm{~d} x+\int_{\Gamma^{\varepsilon}} \alpha_{m} \partial_{\mathbf{n}} u_{m}^{\varepsilon} w_{f} \mathrm{~d} s=0 .
$$

In the case where $w_{f}=0$, for all $w \in \mathrm{V}_{0}^{\varepsilon}$, we get

$$
\int_{\Omega_{m}^{\varepsilon}} \alpha_{m} \Delta u_{m}^{\varepsilon} w_{m} \mathrm{~d} x=0
$$

which implies that

$$
\Delta u_{m}^{\varepsilon}=0 \text { a.e. on } \Omega_{m}^{\varepsilon} .
$$

Clearly, we may deduce from (7.2) and (7.3) that

$$
a_{f}^{\varepsilon}=-\frac{\alpha_{m}}{\alpha_{f} \mu\left(\Omega_{f}^{\varepsilon}\right)} \int_{\Gamma^{\varepsilon}} \partial_{\mathbf{n}} u_{m}^{\varepsilon} x_{1} \mathrm{~d} s \quad \text { and } \quad b_{f}^{\varepsilon}=-\frac{\alpha_{m}}{\alpha_{f} \mu\left(\Omega_{f}^{\varepsilon}\right)} \int_{\Gamma^{\varepsilon}} \partial_{\mathbf{n}} u_{m}^{\varepsilon} x_{2} \mathrm{~d} s .
$$

While in the case where $w_{f}=1$, we find

$$
\int_{\Gamma^{\varepsilon}} \alpha_{m} \partial_{\mathbf{n}} u_{m}^{\varepsilon} \mathrm{d} s=0
$$


According to (7.4) and (7.5), we deduce $u^{\varepsilon}$ satisfies the following problem:

$$
\begin{array}{r}
\alpha_{m} \Delta u_{m}^{\varepsilon}=0 \text { on } \Omega_{m}^{\varepsilon}, \\
u_{m}^{\varepsilon}=u_{f}^{\varepsilon} \text { on } \Gamma^{\varepsilon} \\
u_{f}^{\varepsilon}\left(x_{1}, x_{2}\right)=-\frac{\alpha_{m}}{\alpha_{f} \mu\left(\Omega_{f}^{\varepsilon}\right)}\left(x_{1} \int_{\Gamma^{\varepsilon}} \partial_{\mathbf{n}} u_{m}^{\varepsilon} x_{1} \mathrm{~d} s+x_{2} \int_{\Gamma^{\varepsilon}} \partial_{\mathbf{n}} u_{m}^{\varepsilon} x_{2} \mathrm{~d} s\right) \text { in } \Omega_{f}^{\varepsilon}, \\
\int_{\Gamma^{\varepsilon}} \alpha_{m} \partial_{\mathbf{n}} u_{m}^{\varepsilon} \mathrm{d} s=0 \\
u_{m}^{\varepsilon}=\mathrm{U} \text { on } \Gamma .
\end{array}
$$

\section{Conclusion}

In this paper, we presented an approximation model for a small inclusion in an elastic medium, in antiplane displacement, the thick-point approximation, which consists in approximating the displacement in the inclusion in a finite dimensional space. In order to justify its relevance, we have presented an asymptotic analysis of the initial model, derived from [5], as well as an asymptotic analysis of the approximated model. These two analyses allowed us to derive an error analysis between the initial model and the approximated one and allowed us to distinguish between completely optimal cases and non-optimal cases. Specifically, the first and second degree polynomial approximation was not found optimal in the general case with only a convergence in $\mathcal{O}(\varepsilon)$. Optimality (i.e. $\mathcal{O}\left(\varepsilon^{2}\right)$ and $\mathcal{O}\left(\varepsilon^{3}\right)$ convergence respectively) was obtained with the enrichment of the polynomial spaces with a few solutions to the equations defining the correctors. A notable exception is the case of the circular inclusion where a the optimality is reached without enrichment.

One of the main perspectives of this work, apart from the fact that it should easily extend to the case of plane elasticity as in [5], is to derive optimal numerical methods for the account of small inclusions in media at the lowest computational cost.

\section{Acknowledgments}

We would like to especially thank the company "Manufacture Française des Pneumatiques Michelin" for the financial and technical support.

\section{References}

[1] G. Allaire. Shape optimization by the homogenization method. Volume 146 of Applied Mathematical Sciences, Springer-Verlag, New York (2002).

[2] H. Ammari, H. Kang. Reconstruction of small inhomogeneities from boundary measurements. Springer-Verlag, Berlin Heidelberg (2004).

[3] C. Amrouche, V. Girault, J. Giroire. Weighted Sobolev spaces for LaplaceÕs equation in Rn. J. Math. Pures Appl., 73, (1994), 579-606.

[4] Y. Antipov, P. Schiavone. On the uniformity of stresses inside an inhomogeneity of arbitrary shape Int. J. Eng. Sci., 68, (2003), 299-311. 
[5] M. Arfaoui, M. R. Ben Hassine, M. Moakher, Y. Renard, G. Vial. Multi-scale asymptotic expansion for a small inclusion in elastic media. Journal of Elasticity, 131:207-237, 2018.

[6] E. Beretta, E. Bonnetier, E. Francini, A. L. Mazzucato. An asymptotic formula for the displacement field in the presence of small anisotropic elastic inclusions. Inverse Problems and Imaging, 6, (2012), 1-23.

[7] A. Bendali, K. Lemrabet. The effect of a thin coating on the scattering of a time-harmonic wave for the Helmholtz equation. SIAM J. Appl. Math., 56(6), (1996), 1664-1693.

[8] E. Bonnetier, F. Triki Asymptotics in the presence of inclusions of small volume for a conduction equation: A case with a non-smooth reference potential. Contemp. Math., 494, (2009), 95-107.

[9] H. BRezis Progress in Nonlinear Differential Equations and Their Applications: In memory of Pierre Grisvard Birkhäuser, Boston (1996).

[10] M. Dambrine, G. Vial. Influence of a boundary perforation on a Dirichlet energy Control and Cybernetics, 34(1), (2005), 117-136.

[11] M. Dambrine, G. Vial. A multiscale correction method for local singular perturbations of the boundary. Math. Model. Numer. Anal., 41(1), (2007), 111-127.

[12] A. Ern, J.-L. Guermond. Theory and Practice of Finite Elements. Springer-Verlag, New York (2004).

[13] J. D. Eshelby. The determination of the elastic field of an ellipsoidal inclusion, and related problems. Proceedings of the Royal Society of London. Series A, Mathematical and Physical Sciences, 241, (1957), 376-396.

[14] J. D. Eshelby. The elastic field outside an ellipsoidal inclusion. Proceedings of the Royal Society of London. Series A, Mathematical and Physical Sciences, 252, (1959), 561-569.

[15] A. Fortin, A. Garon. Les Éléments Finis : de la Théorie à la Pratique. Université Laval Ecole Polytechnique de Montréal, Montréal (2011).

[16] G. Geymonat, F. Krasucki, S. Lenci. Mathematical analysis of a bonded joint with a soft thin adhesive. Math. Mech. Solids, 4(2), (1999), 201-225.

[17] D. J. Hansen, C. Poignard, and M. S. Vogelius. Asymptotically precise norm estimates of scattering from a small circular inhomogeneity. Appl. Anal., 86(4), (2007), 433-458.

[18] A. M. ILÕIn. Matching of Asymptotic Expansions of Solutions of Boundary Value Problems. Amer. Math. Soc., Providence RI (1992).

[19] E. KRÖNER. Statistical Modelling. In J. Gittus and J. Zarka, editors, Modelling Small Deformations of Polycrystals, Springer, Dordrecht, the Netherlands (1986), 229-291.

[20] E. KRÖNER. Modified Green Functions in the Theory of Heterogeneous and/or Anisotropic Linearly Elastic Media. In G. J. Weng, M. Taya, and H. Ab, editors Micromechanics and Inhomogeneity: The Toshio Mura 65th Anniversary Volume, Springer, New York, NY (1990), 197-211. 
[21] D. Leguillon, E. SÁnchez-Palencia. Computation of singular solutions in elliptic problems and elasticity. Masson, Paris (2002).

[22] T. Lewinski and J. Sokolowski. Topological derivative for nucleation of non-circular voids. The Neumann problem. In R. Gulliver, W. Littman, R. Triggiani, editors. Differential Geometric Methods in the Control of Partial Differential Equations, volume 268, Amer. Math. Soc., Providence, RI (2000), 341-361.

[23] E. Lignon. Modélisation multi-échelles de nappes fibrés en compression. Ph.D. thesis, Ecole Polytechnique (2011).

[24] J.-L. Lions, E. Magenes. Problèmes aux limites non homogènes et applications. Vol. 1. (French) Travaux et Recherches Mathématiques No17 Dunod, Paris (1968).

[25] S. Li, R. Sauer, G. Wang. A circular inclusion in a finite domain I. The Dirichlet-Eshelby problem. Acta Mechanica, 179(1), (2005), 67-90.

[26] V. Maz'ya, S. Nazarov, B. Plamenevskij. Asymptotic Theory of Elliptic Boundary Value Problems in Singularly Perturbed Domains. Birkhaüser Verlag, Basel (2000).

[27] V. Maz'ya, S. Nazarov, B. Plamenevskij. Asymptotic Theory of Elliptic Boundary Value Problems in Singularly Perturbed Domains. Birkhaüser Verlag, Basel (2000).

[28] P. Mazilu. On the theory of linear elasticity in statically homogeneous media. Rev. Roum. Math. Pur. Appl., 17, (1972), 261-273.

[29] E. SÁnchez-PAlencia. Problèmes de perturbations liés aux phénomènes de conduction à travers des couches minces de grande résistivité. J. Math. Pures Appl., 53(9), (1974), 251-269.

[30] P. Schiavone. Neutrality of the elliptic inhomogeneity in the case of non-uniform loading. IMA journal of applied mathematics, 8, (2003), 161-169.

[31] Y. Sun, Y. Peng. Analytic solutions for the problems of an inclusion of arbitrary shape embedded in a half space. Applied Mathematics and Computation, 140, (2003), 105-113.

[32] G. VIAL. Analyse multi-échelle et conditions aux limites approchées pour un problème avec couche mince dans un domaine coins. PhD thesis Université de Rennes 1 (2003). 